J. DIFFERENTIAL GEOMETRY

72 (2006) 73-112

\title{
GENERALIZATIONS OF MCSHANE'S IDENTITY TO HYPERBOLIC CONE-SURFACES
}

\author{
Ser Peow Tan, Yan Loi Wong \& Ying Zhang
}

\begin{abstract}
We generalize McShane's identity for the length series of simple closed geodesics on a cusped hyperbolic surface [19] to a general identity for hyperbolic cone-surfaces (with all cone angles $\leq \pi$ ), possibly with cusps and/or geodesic boundary. The general identity is obtained by studying gaps formed by simple-normal geodesics emanating from a distinguished cone point, cusp or boundary geodesic. In particular, by applying the generalized identity to the quotient orbifolds of a hyperbolic one-cone/one-hole torus by its elliptic involution and of a hyperbolic closed genus two surface by its hyperelliptic involution, we obtain general Weierstrass identities for the one-cone/one-hole torus, and an identity for the genus two surface, which are also obtained by McShane using different methods in $[\mathbf{2 0}],[\mathbf{2 2}]$ and $[\mathbf{2 1}]$. We also give an interpretation of the general identity in terms of complex lengths of the cone points, cusps and geodesic boundary components.
\end{abstract}

\section{Introduction}

Greg McShane discovered in his thesis [18] the following striking identity.

Theorem 1.1 (McShane [18]). In a hyperbolic torus $T$,

$$
\sum_{\gamma} \frac{1}{1+\exp |\gamma|}=\frac{1}{2}
$$

where the sum extends over all simple closed geodesics on $T$ and where $|\gamma|$ denotes the length of $\gamma$ in the given hyperbolic structure.

Here, by a hyperbolic torus, we mean a once punctured torus equipped with a complete hyperbolic structure of finite area, and throughout the paper, we shall always use $|\gamma|$ to denote the hyperbolic length of $\gamma$ if

The authors gratefully acknowledge support of the National University of Singapore academic research grant R-146-000-056-112. The third author was partially supported by the National Key Basic Research Fund (China) G1999075104 while writing this paper.

Received 10/02/2004. 
$\gamma$ is a (generalized) simple closed geodesic or a simple geodesic arc on a hyperbolic (cone-)surface. All surfaces we consider in this paper are assumed to be connected and orientable.

Later, in [19], McShane extended his identity to more general hyperbolic surfaces with cusps as follows.

Theorem 1.2 (McShane [19]). In a finite area hyperbolic surface $M$ with cusps and without boundary,

$$
\sum \frac{1}{1+\exp \frac{1}{2}(|\alpha|+|\beta|)}=\frac{1}{2},
$$

where the sum is taken over all unordered pairs of simple closed geodesics $\alpha, \beta$ (where $\alpha$ or $\beta$ might be a cusp treated as a simple closed geodesic of length 0$)$ on $M$ such that $\alpha, \beta$ bound with a distinguished cusp point an embedded pair of pants on $M$.

Note that Theorem 1.1 can be regarded as a special case of Theorem 1.2 where $\alpha, \beta$ are the same for each pair $\alpha, \beta$.

In [20], McShane demonstrated three other closely related identities for the lengths of simple closed geodesics in each of the three Weierstrass classes on a hyperbolic torus. Recall that a hyperbolic torus $T$ has three Weierstrass points which are the fixed points of the unique elliptic involution which maps each simple closed geodesic on $T$ onto itself with orientation reversed, and for a Weierstrass point $x$ on $T$, the simple closed geodesics in the Weierstrass class which is dual to $x$ are precisely all the simple closed geodesics on $T$ which do not pass through $x$.

Theorem 1.3 (McShane $[\mathbf{2 0}]$ ). In a hyperbolic torus $T$,

$$
\sum_{\gamma \in \mathcal{A}} \sin ^{-1}\left(\frac{1}{\cosh \frac{1}{2}|\gamma|}\right)=\frac{\pi}{2},
$$

where the sum is taken over all simple closed geodesics in a given Weierstrass class $\mathcal{A}$ on $T$.

On the other hand, Bowditch gave an alternative proof of Theorem 1.1 using Markoff triples [7] and extended the identity in Theorem 1.1 to the case of quasi-Fuchsian representations of the torus group [9] as well as to the case of hyperbolic once punctured torus bundles [8]. There are also some other generalizations along these directions, by Makoto Sakuma and his co-workers, see $[\mathbf{1}],[\mathbf{2}],[\mathbf{2 4}]$.

In this paper, we further generalize McShane's identity as in Theorem 1.2 to the cases of hyperbolic cone-surfaces possibly with cusps and/or geodesic boundary. (See for example [11] for basic facts on cone-manifolds.) We assume that all cone points have cone angle $\leq \pi$ (except for the one-cone torus where we allow the cone angle up to $2 \pi$ ). The ideas are related in spirit to those in $[\mathbf{3}]$ while the method of proof 
follows closely that of McShane's in [19]. The key points are that the assumption that all cone angles are $\leq \pi$ implies that all non-peripheral simple closed curves are essentially realizable as simple geodesics in their free (relative) homotopy classes; and that the Birman-Series result [6] on the sparsity of simple geodesics carries over to this case, in particular to simple geodesic rays emanating (normally) from a fixed boundary component. It should be noted that our result shows that the assumption of discreteness of the holonomy group is unnecessary, and that it gives identities for all hyperbolic orbifold surfaces. We also show how the result can be formulated in terms of complex lengths (Theorem 1.16) even though the situation we consider here is real. This is particularly useful, and is explored further in [29] and [28], where we show how this approach allows us to generalize McShane's identity to identities for marked classical Schottky groups, and how Bowditch's study of Markoff maps in [7] can be generalized as well to give identities for certain generalized Markoff maps. (See also [13] for related work on generalized Markoff triples.) This also leads to generalizations of Bowditch's interpretation [8] of McShane's identity for complete hyperbolic 3-manifolds which are once punctured torus bundles over the circle to identities for the hyperbolic 3-manifolds obtained by hyperbolic Dehn surgery on such manifolds. This is explored in [28], and should tie up nicely with the work of Sakuma in [24], and Akiyoshi-Miyachi-Sakuma in $[\mathbf{1}]$ and $[\mathbf{2}]$.

To state the most general form of our generalized McShane's identities, we need to introduce some new terminology. However, to let the reader get the flavor of the generalized identities, we first state the corresponding generalizations of Theorems 1.1 and 1.2.

Theorem 1.4. Let $T$ be either a hyperbolic one-cone torus where the single cone point has cone angle $\theta \in(0,2 \pi)$ or a hyperbolic one-hole torus where the single boundary geodesic has length $l>0$. Then, we have respectively

$$
\begin{gathered}
\sum_{\gamma} 2 \tan ^{-1}\left(\frac{\sin \frac{\theta}{2}}{\cos \frac{\theta}{2}+\exp |\gamma|}\right)=\frac{\theta}{2}, \\
\sum_{\gamma} 2 \tanh ^{-1}\left(\frac{\sinh \frac{l}{2}}{\cosh \frac{l}{2}+\exp |\gamma|}\right)=\frac{l}{2},
\end{gathered}
$$

where the sum in either case extends over all simple closed geodesics on $T$.

Theorem 1.5. Let $M$ be a compact hyperbolic cone-surface with a single cone point of cone angle $\theta \in(0, \pi]$ and without boundary or let $M$ be a compact hyperbolic surface with a single boundary geodesic having 
length $l>0$. Then, we have respectively

$$
\begin{gathered}
\sum 2 \tan ^{-1}\left(\frac{\sin \frac{\theta}{2}}{\cos \frac{\theta}{2}+\exp \frac{|\alpha|+|\beta|}{2}}\right)=\frac{\theta}{2}, \\
\sum 2 \tanh ^{-1}\left(\frac{\sinh \frac{l}{2}}{\cosh \frac{l}{2}+\exp \frac{|\alpha|+|\beta|}{2}}\right)=\frac{l}{2},
\end{gathered}
$$

where the sum in either case extends over all unordered pairs of simple closed geodesics on $M$ which bound with the cone point (respectively, the boundary geodesic) an embedded pair of pants.

For the purposes of this paper, we make the following definition.

Definition 1.6. A compact hyperbolic cone-surface $M$ is a compact (topological) surface $M$ with hyperbolic cone structure where each boundary component is a smooth simple closed geodesic and where there are a finite number of interior points which form all the cone points and cusps. Its geometric boundary, denoted $\Delta M$, is the union of all cusps, cone points and geodesic boundary components. (Note that $\Delta M$ is different from the usual topological boundary $\partial M$ when there are cusps or cone points.) Thus, a geometric boundary component is either a cusp, a cone point, or a boundary geodesic. The geometric interior of $M$ is $M-\Delta M$.

In this paper, we consider a compact hyperbolic cone-surface $M=$ $M\left(\Delta_{0} ; k, \Theta, L\right)$ with $k$ cusps $C_{1}, C_{2}, \ldots, C_{k}$, with $m$ cone points $P_{1}, P_{2}$, $\ldots, P_{m}$, where the cone angle of $P_{i}$ is $\theta_{i} \in(0, \pi], i=1,2, \ldots, m$, and with $n$ geodesic boundary components $B_{1}, B_{2}, \ldots, B_{n}$, where the length of $B_{i}$ is $l_{i}>0, i=1,2, \ldots, n$, together with an extra distinguished geometric boundary component $\Delta_{0}$. Thus, $\Delta_{0}$ is either a cusp $C_{0}$ or a cone point $P_{0}$ of cone angle $\theta_{0} \in(0, \pi]$ or a geodesic boundary component $B_{0}$ of length $l_{0}>0$. Note that in the above notation $\Theta=\left(\theta_{1}, \theta_{2}, \ldots, \theta_{m}\right)$ and $L=\left(l_{1}, l_{2}, \ldots, l_{n}\right)$. We exclude the case where $M$ is a geometric pair of pants for we have only trivial identities in that case.

We allow that some (even all) of the cone angles $\theta_{i}$ are equal to $\pi, i=0,1, \ldots, m$; these are often cases of particular interest. However, for clarity of exposition, quite often in proofs/statements of lemmas/theorems, we shall first consider the case where all the cone angles are less than $\pi$ and then point out the addenda that should be made when there are angle $\pi$ cone points. The advantage of this assumption of strict inequality is that every non-trivial, non-peripheral simple closed curve on such $M$ can be realized as a (smooth) simple closed geodesic in its free homotopy class in the geometric interior of $M$ under the given hyperbolic cone-structure (see Section 5 for the proof of this statement).

We call a simple closed curve on $M$ peripheral if it is freely homotopic on $M$ to a geometric boundary component of $M$. 
Definition 1.7. By a generalized simple closed geodesic on $M$, we mean either

(i) a simple closed geodesic in the geometric interior of $M$; or

(ii) a degenerate simple closed geodesic which is the double of a simple geodesic arc in the geometric interior of $M$ connecting two angle $\pi$ cone points; or

(iii) a geometric boundary component, that is, a cusp or a cone point or a boundary geodesic.

In particular, generalized simple closed geodesics of the first two kinds are called interior generalized simple closed geodesics.

For each unordered pair of generalized simple closed geodesics $\alpha, \beta$ which bound with $\Delta_{0}$ an embedded geometric pair of pants, we shall define a normalized gap function $\operatorname{Gap}^{\prime}\left(\Delta_{0} ; \alpha, \beta\right)$ when $\Delta_{0}$ is a cusp (case 0 of Section 4), and a gap function $\operatorname{Gap}\left(\Delta_{0} ; \alpha, \beta\right)$ when $\Delta_{0}$ is a cone point or a boundary geodesic (cases 1 and 2 of Section 4 ). The definitions are deferred to Section 4 as they are somewhat complicated and depend on several subcases.

Now, we are in a position to state the most general (real) form of our generalization of McShane's identity.

Theorem 1.8. Let $M$ be a compact hyperbolic cone-surface with all cone angles in $(0, \pi]$. Then, one has either

$$
\sum \operatorname{Gap}\left(\Delta_{0} ; \alpha, \beta\right)=\frac{\theta_{0}}{2},
$$

when $\Delta_{0}$ is a cone point of cone angle $\theta_{0}$; or

$$
\sum \operatorname{Gap}\left(\Delta_{0} ; \alpha, \beta\right)=\frac{l_{0}}{2},
$$

when $\Delta_{0}$ is a boundary geodesic of length $l_{0}$; or

$$
\sum \operatorname{Gap}^{\prime}\left(\Delta_{0} ; \alpha, \beta\right)=\frac{1}{2},
$$

when $\Delta_{0}$ is a cusp; where, in each case, the sum is taken over all unordered pairs of generalized simple closed geodesics $\alpha, \beta$ on $M$ which bound with $\Delta_{0}$ an embedded pair of pants.

\section{Remark 1.9.}

(i) In the case of the hyperbolic one-cone torus, the theorem holds for $\theta_{0} \in(0,2 \pi)$.

(ii) In the special cases where the geometric boundary $\Delta M$ is a single cone point or a single boundary geodesic, Theorem 1.8 gives all the previously stated generalized identities in Theorems 1.4 and 1.5 .

(iii) We use the notation Gap' for the function in the cusp case (that is, $\Delta_{0}$ is a cusp) as it is the limit case of the other cases as the cone angle $\theta_{0}$ or the boundary geodesic length $l_{0}$ approaches 0 , 
and so can be considered as the formal derivative of the function Gap used in the first two cases, evaluated at $\theta_{0}=0$ or $l_{0}=0$. The identity in the cusp case can indeed be derived from the first order infinitesimal of the identities of the other cases.

It is also interesting to note that McShane's Weierstrass identities can be deduced as special cases of our general Theorem 1.8 by applying the theorem to the quotient of a hyperbolic one-cone/one-hole torus by its elliptic involution and then lifting back to the torus. Thus, we have the following generalized Weierstrass identities.

Corollary 1.10. Let $T$ be either a hyperbolic one-cone torus where the single cone point has cone angle $\theta \in(0,2 \pi)$ or a hyperbolic one-hole torus where the single boundary geodesic has length $l>0$. Then, we have respectively

$$
\begin{aligned}
& \sum_{\gamma \in \mathcal{A}} \tan ^{-1}\left(\frac{\cos \frac{\theta}{4}}{\sinh \frac{|\gamma|}{2}}\right)=\frac{\pi}{2}, \\
& \sum_{\gamma \in \mathcal{A}} \tan ^{-1}\left(\frac{\cosh \frac{l}{4}}{\sinh \frac{|\gamma|}{2}}\right)=\frac{\pi}{2},
\end{aligned}
$$

where the sum in either case is taken over all the simple closed geodesics $\gamma$ in a given Weierstrass class $\mathcal{A}$.

McShane's original Weierstrass identity (1.3) then corresponds to the case $\theta=0$ or $l=0$ in the above two identities, noticing that

$$
\tan ^{-1}\left(\frac{1}{\sinh \frac{|\gamma|}{2}}\right)=\sin ^{-1}\left(\frac{1}{\cosh \frac{|\gamma|}{2}}\right) .
$$

As further corollaries, there are the following weaker but neater identities, each of which is obtained by summing the three Weierstrass identities in the corresponding case.

Corollary 1.11. Let $T$ be a hyperbolic torus whose geometric boundary is either a single cusp, a single cone point of cone angle $\theta \in(0,2 \pi)$, or a single boundary geodesic of length $l>0$. Then, we have respectively

$$
\begin{aligned}
& \sum_{\gamma} \tan ^{-1}\left(\frac{1}{\sinh \frac{|\gamma|}{2}}\right)=\frac{3 \pi}{2}, \\
& \sum_{\gamma} \tan ^{-1}\left(\frac{\cos \frac{\theta}{4}}{\sinh \frac{|\gamma|}{2}}\right)=\frac{3 \pi}{2}, \\
& \sum_{\gamma} \tan ^{-1}\left(\frac{\cosh \frac{l}{4}}{\sinh \frac{|\gamma|}{2}}\right)=\frac{3 \pi}{2},
\end{aligned}
$$


where the sum in each case is taken over all the simple closed geodesics $\gamma$ on $T$.

Remark 1.12. The identity (1.15) was also obtained by McShane [21] using Wolpert's variation of length method. It seems likely his method can be extended to prove some of the other identities as well.

Similarly, for a genus two closed hyperbolic surface $M$, one can consider the (six) identities on the quotient surface $M / \eta$ where $\eta$ is the unique hyperelliptic involution on $M$ (note that $M / \eta$ is a closed hyperbolic orbifold of genus 0 with six cone angle $\pi$ points, and we may choose any one of these cone points to be the distinguished geometric boundary component) and reinterpret them as Weierstrass identities on the original surface $M$ (see also McShane [22] where the Weierstrass identities were obtained directly). Combining all the six Weierstrass identities for $M$, we then have the following very neat identity.

Theorem 1.13. Let $M$ be a genus two closed hyperbolic surface. Then

$$
\sum \tan ^{-1} \exp \left(-\frac{|\alpha|}{4}-\frac{|\beta|}{2}\right)=\frac{3 \pi}{2},
$$

where the sum is taken over all ordered pairs $(\alpha, \beta)$ of disjoint simple closed geodesics on $M$ such that $\alpha$ is separating and $\beta$ is non-separating.

Remark 1.14. This is the only case that we know of where McShane's identity extends in a nice way to a closed surface.

We observe that the above identity for closed genus two surface $M$ also extends to quasi-Fuchsian representations of $\pi_{1}(M)$. The space of quasi-Fuchsian representations is well studied, and known to be contractible and homeomorphic to $\mathbf{R}^{12 g-12}$ for a closed surface of genus $g$. For more details of the geometry and topology of the space of quasiFuchsian representations, see for example [5] and [17].

Let $\rho: \pi_{1}(M) \rightarrow \operatorname{PSL}(2, \mathbf{C})$ be a quasi-Fuchsian representation. For each essential simple closed curve $\gamma$, let $l_{\rho}(\gamma) \in \mathbf{C}$, with positive real part, be defined by

$$
\cosh \left(l_{\rho}(\gamma)\right)=\frac{1}{2} \operatorname{tr}^{2} \rho([\gamma])-1
$$

(where $[\gamma] \in \pi_{1}(M)$ is any homotopy class in the conjugacy classes up to inversion in $\pi_{1}(M)$ corresponding to $\gamma$ ) and by analytic continuation from the space of Fuchsian representations to the space of quasiFuchsian representations. This is well-defined since the quasi-Fuchsian space is contractible and each $\rho([\gamma])$ is a loxodromic or hyperbolic element. Note that $l_{\rho}(\gamma)$ is also called the complex length of $\rho([\gamma])$. 
Addendum 1.15. For a quasi-Fuchsian representation $\rho: \pi_{1}(M) \rightarrow$ $\operatorname{PSL}(2, \mathbf{C})$ for the closed genus two surface $M$, we have

$$
\sum \tan ^{-1} \exp \left(-\frac{l_{\rho}(\alpha)}{4}-\frac{l_{\rho}(\beta)}{2}\right)=\frac{3 \pi}{2},
$$

where the sum, which converges absolutely, is taken over all the ordered pairs $[\alpha],[\beta]$ of free homotopy classes up to inversion of disjoint unoriented essential simple closed curves $\alpha, \beta$ on $M$ such that $\alpha$ is separating and $\beta$ is non-separating.

Note that here and in the rest of the paper, the function $\tan ^{-1}$ : $\mathbf{C} \backslash\{ \pm i\} \rightarrow \mathbf{C}$ is defined by

$$
\tan ^{-1} u=\frac{1}{2 i} \log \frac{1+u i}{1-u i}
$$

where the log function assumes its main branch value, i.e., with imaginary part in $(-\pi, \pi]$. Similarly, the function $\tanh ^{-1}: \mathbf{C} \backslash\{ \pm 1\} \rightarrow \mathbf{C}$ is defined by

$$
\tanh ^{-1} u=\frac{1}{2} \log \frac{1+u}{1-u},
$$

where again the log function assumes its main branch value. Hence, it is always true that

$$
i \tan ^{-1} u=\tanh ^{-1}(u i) \quad \text { for } \quad u \neq \pm i .
$$

It is easy to check that if $w \in \mathbf{C}$ lies in the open unit disk, then

$$
\frac{1+w}{1-w} \notin \mathbf{R}_{\leq 0} .
$$

Thus, the above defined functions $\tan ^{-1}$ and $\tanh ^{-1}$ are analytic in the open unit disk. Now, we have

$$
\left|\exp \left(-\frac{l_{\rho}(\alpha)}{4}-\frac{l_{\rho}(\beta)}{2}\right)\right|<1
$$

since $\Re l_{\rho}(\alpha), \Re l_{\rho}(\beta)>0$. It follows that each summand on the left-hand side of (1.18) is an analytic function on the quasi-Fuchsian space of $M$ with no need for analytic continuation of the $\tan ^{-1}$ function involved. This explains why (1.18) is an identity without the modulo $\pi$ condition.

In the statement of Theorem 1.8, we did not write down the explicit expression for the gap functions due to their "case by case" nature as can be seen in Section 4. The cone points and boundary geodesics as geometric boundary components seem to have different roles in the series in the generalized identities, resulting in the identities being somewhat ad-hoc and not unified.

This difference can, however, be removed by assigning purely imaginary length to a cone point as a geometric boundary component. More precisely, for each generalized simple closed geodesic $\delta$, we define its complex length $|\delta|$ as: $|\delta|=0$ if $\delta$ is a cusp; $|\delta|=\theta i$ if $\delta$ is a cone 
point of angle $\theta \in(0, \pi]$; and $|\delta|=l$ if $\delta$ is a boundary geodesic or an interior generalized simple closed geodesic of length $l>0$. Then, we can reformulate the generalized McShane's identities in Theorem 1.8 as follows, the case by case analysis is now replaced by just two fairly simple elementary functions.

Theorem 1.16. Let $M$ be a compact hyperbolic cone-surface with all cone angles in $(0, \pi]$, and let all its geometric boundary components be $\Delta_{0}, \Delta_{1}, \ldots, \Delta_{N}$ with complex lengths $L_{0}, L_{1}, \ldots, L_{N}$ respectively. Then

$$
\begin{aligned}
& \sum_{\alpha, \beta} 2 \tanh ^{-1}\left(\frac{\sinh \frac{L_{0}}{2}}{\cosh \frac{L_{0}}{2}+\exp \frac{|\alpha|+|\beta|}{2}}\right) \\
& \quad+\sum_{j=1}^{N} \sum_{\beta} \tanh ^{-1}\left(\frac{\sinh \frac{L_{0}}{2} \sinh \frac{L_{j}}{2}}{\cosh \frac{|\beta|}{2}+\cosh \frac{L_{0}}{2} \cosh \frac{L_{j}}{2}}\right)=\frac{L_{0}}{2},
\end{aligned}
$$

if $\Delta_{0}$ is a cone point or a boundary geodesic; and

$$
\sum_{\alpha, \beta} \frac{1}{1+\exp \frac{|\alpha|+|\beta|}{2}}+\sum_{j=1}^{N} \sum_{\beta} \frac{1}{2} \frac{\sinh \frac{L_{j}}{2}}{\cosh \frac{|\beta|}{2}+\cosh \frac{L_{j}}{2}}=\frac{1}{2},
$$

if $\Delta_{0}$ is a cusp; where, in either case, the first sum is taken over all unordered pairs of generalized simple closed geodesics $\alpha, \beta$ on $M$ which bound with $\Delta_{0}$ an embedded pair of pants on $M$ (note that one of $\alpha, \beta$ might be a geometric boundary component) and the sub-sum in the second sum is taken over all interior simple closed geodesics $\beta$ which bounds with $\Delta_{j}$ and $\Delta_{0}$ an embedded pair of pants on $M$.

Furthermore, each series in (1.22) and (1.23) converges absolutely.

Remark 1.17. Note that the absolute convergence of the various series in (1.22) and (1.23) is not obvious, as the summands in the series are no longer all real and positive. For example, when $\Delta_{0}$ is a boundary geodesic and some of the $\Delta_{j}$ 's are cone points, it is easily checked that terms in the first series of the identity (1.22) where one of $\alpha$ or $\beta$ is a cone geometric boundary have non-zero real and imaginary parts. We shall discuss this in more detail at the end of Section 10. The point of view is also useful in generalizing the identities to classical Schottky groups, see [29].

Additional Remark. We were informed while writing this paper by Makoto Sakuma and Caroline Series of the recent striking results of Maryam Mirzakhani [23] where she had generalized McShane's identities to hyperbolic surfaces with boundary and used it to calculate the Weil-Petersson volumes of the corresponding moduli spaces. There is obviously an overlap of her results with ours, in particular, the identities she obtains are equivalent to ours in the case of hyperbolic surfaces with boundary (see Section 10 for further explanations). Her expressions in 
terms of the log function seem particularly well suited to her purpose of calculating the Weil-Petersson volumes. In fact, as already observed by her in [23], her results should extend to the cone-manifold case. Hence, combined with our results, we get extensions of her formulae for the Weil-Petersson volumes to the volumes of the moduli spaces of compact hyperbolic cone-surfaces with all cone angles bounded above by $\pi$, where a cone point of angle $\theta$ is regarded as a geometric boundary component with purely imaginary length $\theta i$.

\section{Acknowledgements}

We would like to thank Caroline Series for helpful conversations; Makoto Sakuma for his encouragement to write up our results on the cone-manifold case (during conversations with the first named author at the Isaac Newton Institute in August, 2003) and also for bringing to our attention the recent works of McShane [21] and Mirzakhani [23]; and Greg McShane for helpful e-mail correspondence and also for bringing our attention to $[\mathbf{2 2}]$. We would also like to thank the referee for the many useful comments and suggestions. The third named author would like to thank Professors Weiyue Ding, Hongyu Wang and Youde Wang for their encouragement and concerns.

\section{The organization of the rest of the paper}

The rest of the paper is organized as follows. In Section 4, we define the gap functions used in Theorem 1.8 for the various cases. In Section 5 , we deal with the problem of realization of simple closed curves by geodesics, and show that the assumption that all cone angles are less than or equal to $\pi$ is essential. In Section 6, we analyze the so-called $\Delta_{0}$-geodesics, that is, the geodesics starting/emanating orthogonally from $\Delta_{0}$, and determine all the gaps between all simple-normal $\Delta_{0^{-}}$ geodesics, that is, the simple $\Delta_{0}$-geodesics which either never hit any geometric boundary components or terminate at geometric boundary components orthogonally. In Section 7 , we calculate the gap function which is the width of a combined gap measured suitably. In Section 8, we show that the Birman-Series Theorem (which states that the point set of all complete geodesics with bounded self intersection numbers on a compact hyperbolic surface has Hausdorff dimension 1) extends to the case of compact hyperbolic cone-surfaces with all cone angles less than or equal to $\pi$. In Section 9, we give proofs of the other theorems, except that of Theorem 1.16, which is deferred to the last section. Finally in Section 10, we restate the complexified generalized McShane's identity (1.22) (Theorem 1.16) using two functions of complex variables and hence, unify the somewhat unattractive "case-by-case" definition of the gap functions. We interpret the geometric meanings of the complexified 
summands in the complexified generalized McShane's identity and prove the absolute convergence of the complexified series in it by a simple use of the Birman-Series arguments in [6].

\section{Defining the Gap functions}

In this section, for a compact hyperbolic cone-surface $M=M\left(\Delta_{0} ; k\right.$, $\Theta, L)$ with all cone angles $\leq \pi$ we define the gap function $\operatorname{Gap}\left(\Delta_{0} ; \alpha, \beta\right)$ (when $\Delta_{0}$ is a cone point or a boundary geodesic) and the normalized gap function $\operatorname{Gap}^{\prime}\left(\Delta_{0} ; \alpha, \beta\right)$ (when $\Delta_{0}$ is a cusp) where $\alpha, \beta$ are generalized simple closed geodesics on $M$ which bound with $\Delta_{0}$ a geometric pair of pants. These functions essentially measure the (combined) gaps in the complement of the set of complete simple geodesics that emanate normally from $\Delta_{0}$, as explained in Section 6 and computed in Section 7 .

Throughout the paper, we use $|\alpha|$ to denote the length of $\alpha$ when $\alpha$ is an interior generalized simple closed geodesic or a boundary geodesic. In particular, when $\alpha$ is a degenerate simple closed geodesic (that is, the double cover of a simple geodesic arc which connects two angle $\pi$ cone points), its length $|\alpha|$ is defined as twice the length of the simple geodesic arc that it covers.

Recall that an interior generalized simple closed geodesic is either a simple closed geodesic in the geometric interior of $M$ or a degenerate simple closed geodesic on $M$ which is the double cover of a simple geodesic arc which connects two angle $\pi$ cone points.

Case 0. $\Delta_{0}$ is a cusp.

Subcase 0.1. Both $\alpha$ and $\beta$ are interior generalized simple closed geodesics.

In this case,

$$
\operatorname{Gap}^{\prime}\left(\Delta_{0} ; \alpha, \beta\right)=\frac{1}{1+\exp \frac{1}{2}(|\alpha|+|\beta|)} .
$$

Subcase 0.2 . One of $\alpha, \beta$, say $\alpha$, is a boundary geodesic and the other, $\beta$, is an interior generalized simple closed geodesic.

In this case,

$$
\operatorname{Gap}^{\prime}\left(\Delta_{0} ; \alpha, \beta\right)=\frac{1}{2}-\frac{1}{2} \frac{\sinh \frac{|\beta|}{2}}{\cosh \frac{|\alpha|}{2}+\cosh \frac{|\beta|}{2}} .
$$

Subcase 0.3. One of $\alpha, \beta$, say $\alpha$, is a cone point of cone angle $\varphi \in(0, \pi]$ and the other, $\beta$, is an interior generalized simple closed geodesic.

In this case,

$$
\operatorname{Gap}^{\prime}\left(\Delta_{0} ; \alpha, \beta\right)=\frac{1}{2}-\frac{1}{2} \frac{\sinh \frac{|\beta|}{2}}{\cos \frac{\varphi}{2}+\cosh \frac{|\beta|}{2}} .
$$


Subcase 0.4. One of $\alpha, \beta$, say $\alpha$, is also a cusp and the other, $\beta$, is an interior generalized simple closed geodesic.

In this case,

$$
\operatorname{Gap}^{\prime}\left(\Delta_{0} ; \alpha, \beta\right)=\frac{1}{2}-\frac{1}{2} \frac{\sinh \frac{|\beta|}{2}}{1+\cosh \frac{|\beta|}{2}}=\frac{1}{1+\exp \frac{1}{2}|\beta|},
$$

which is the common value of $\operatorname{Gap}\left(\Delta_{0} ; \alpha, \beta\right)$ in Subcases 0.1 through 0.3 when $|\alpha|=0$.

Case 1. $\Delta_{0}$ is a cone point of cone angle $\theta \in(0, \pi]$.

Subcase 1.1. Both $\alpha$ and $\beta$ are interior generalized simple closed geodesics.

In this case,

$$
\operatorname{Gap}\left(\Delta_{0} ; \alpha, \beta\right)=2 \tan ^{-1}\left(\frac{\sin \frac{\theta}{2}}{\cos \frac{\theta}{2}+\exp \frac{|\alpha|+|\beta|}{2}}\right) .
$$

Subcase 1.2. One of $\alpha, \beta$, say $\alpha$, is a boundary geodesic and the other, $\beta$, is an interior generalized simple closed geodesic.

In this case,

$$
\operatorname{Gap}\left(\Delta_{0} ; \alpha, \beta\right)=\frac{\theta}{2}-\tan ^{-1}\left(\frac{\sin \frac{\theta}{2} \sinh \frac{|\beta|}{2}}{\cosh \frac{|\alpha|}{2}+\cos \frac{\theta}{2} \cosh \frac{|\beta|}{2}}\right)
$$

Subcase 1.3. One of $\alpha, \beta$, say $\alpha$, is a cone point of cone angle $\varphi \in(0, \pi]$ and the other, $\beta$, is an interior generalized simple closed geodesic.

In this case,

$$
\operatorname{Gap}\left(\Delta_{0} ; \alpha, \beta\right)=\frac{\theta}{2}-\tan ^{-1}\left(\frac{\sin \frac{\theta}{2} \sinh \frac{|\beta|}{2}}{\cos \frac{\varphi}{2}+\cos \frac{\theta}{2} \cosh \frac{|\beta|}{2}}\right) .
$$

Note that there is no gap when $\theta=\varphi=\pi$.

Subcase 1.4. One of $\alpha, \beta$, say $\alpha$, is a cusp and the other, $\beta$, is an interior generalized simple closed geodesic.

In this case,

$$
\begin{aligned}
\operatorname{Gap}\left(\Delta_{0} ; \alpha, \beta\right) & =2 \tan ^{-1}\left(\frac{\sin \frac{\theta}{2}}{\cos \frac{\theta}{2}+\exp \frac{|\beta|}{2}}\right) \\
& =\frac{\theta}{2}-\tan ^{-1}\left(\frac{\sin \frac{\theta}{2} \sinh \frac{|\beta|}{2}}{1+\cos \frac{\theta}{2} \cosh \frac{|\beta|}{2}}\right),
\end{aligned}
$$

which is the common value of $\operatorname{Gap}\left(\Delta_{0} ; \alpha, \beta\right)$ in Subcases 1.1 through 1.3 when $|\alpha|=0$.

Case 2. $\Delta_{0}$ is a boundary geodesic of length $l>0$. 
Subcase 2.1. Both $\alpha$ and $\beta$ are interior generalized simple closed geodesics.

In this case,

$$
\operatorname{Gap}\left(\Delta_{0} ; \alpha, \beta\right)=2 \tanh ^{-1}\left(\frac{\sinh \frac{l}{2}}{\cosh \frac{l}{2}+\exp \frac{|\alpha|+|\beta|}{2}}\right) .
$$

Subcase 2.2. One of $\alpha, \beta$, say $\alpha$, is a boundary geodesic and the other, $\beta$, is an interior generalized simple closed geodesic.

In this case,

$$
\operatorname{Gap}\left(\Delta_{0} ; \alpha, \beta\right)=\frac{l}{2}-\tanh ^{-1}\left(\frac{\sinh \frac{l}{2} \sinh \frac{|\beta|}{2}}{\cosh \frac{|\alpha|}{2}+\cosh \frac{l}{2} \cosh \frac{|\beta|}{2}}\right) .
$$

Subcase 2.3. One of $\alpha, \beta$, say $\alpha$, is a cone point of cone angle $\varphi \in(0, \pi]$ and the other, $\beta$, is an interior generalized simple closed geodesic.

In this case,

$$
\operatorname{Gap}\left(\Delta_{0} ; \alpha, \beta\right)=\frac{l}{2}-\tanh ^{-1}\left(\frac{\sinh \frac{l}{2} \sinh \frac{|\beta|}{2}}{\cos \frac{\varphi}{2}+\cosh \frac{l}{2} \cosh \frac{|\beta|}{2}}\right) .
$$

Subcase 2.4. One of $\alpha, \beta$, say $\alpha$, is a cusp and the other, $\beta$, is an interior generalized simple closed geodesic.

In this case,

$$
\begin{aligned}
\operatorname{Gap}\left(\Delta_{0} ; \alpha, \beta\right) & =2 \tanh ^{-1}\left(\frac{\sinh \frac{l}{2}}{\cosh \frac{l}{2}+\exp \frac{|\beta|}{2}}\right) \\
& =\frac{l}{2}-\tanh ^{-1}\left(\frac{\sinh \frac{l}{2} \sinh \frac{|\beta|}{2}}{1+\cosh \frac{l}{2} \cosh \frac{|\beta|}{2}}\right),
\end{aligned}
$$

which is the common value of $\operatorname{Gap}\left(\Delta_{0} ; \alpha, \beta\right)$ in Subcases 2.1 through 2.3 when $|\alpha|=0$.

\section{Realizing simple curves by geodesics on hyperbolic cone-surfaces}

In this section, we consider the problem of realizing essential simple curves in their free (relative) homotopy classes by geodesics on a compact hyperbolic cone-surface $M$ with all cone angles smaller than $\pi$. We show that each essential simple closed curve in the geometric interior of $M$ can be realized uniquely in its free homotopy class (where the homotopy takes place in the geometric interior of $M$ ) as either a geometric boundary component or a simple closed geodesic in the geometric interior of $M$. We also show that each essential simple arc which connects geometric boundary components of $M$ can be realized uniquely in its free relative homotopy class (where the homotopy takes place in the 
geometric interior of $M$ and the endpoints slide on the same geometric boundary components) as a simple geodesic arc which is normal to the geometric boundary components involved. We also make addenda for the cases when there are angle $\pi$ cone points.

Theorem 5.1. Let $M$ be a compact hyperbolic cone-surface with all cone angles less than $\pi$.

(i) If $c$ is an essential non-peripheral simple closed curve in the geometric interior of $M$, then there is a unique simple closed geodesic in the free homotopy class of $c$ in the geometric interior of $M$.

(ii) If $c$ is an essential simple arc which connects geometric boundary components, then there is a unique simple normal geodesic arc in the free relative homotopy class of $c$ in the geometric interior of $M$ with endpoints varying on the respective geometric boundary components.

Addendum 5.2. Suppose in addition that $M$ has some cone angles equal to $\pi$.

(i) In Theorem 5.1(i), if the simple closed curve $c$ bounds with two angle $\pi$ cone points an embedded pair of pants, then the geodesic realization for $c$ is the double cover of the simple geodesic arc which connects these two angle $\pi$ cone points and is homotopic (relative to boundary) to a simple arc lying wholly in the pair of pants;

(ii) In Theorem 5.1(ii), if the simple arc $c$ connects a geometric boundary component $\Delta$ to itself and bounds together with $\Delta$ and an angle $\pi$ cone point $P$ an embedded cylinder, then the geodesic realization for $c$ is the double cover of the normal simple geodesic arc which connects $\Delta$ to $P$ and is homotopic (relative to boundary) to a simple arc lying wholly in the cylinder.

The simple geodesic in Theorem 5.1 and Addendum 5.2 is called the geodesic realization of the given simple curve in the respective homotopy class.

The proof is a well-known use of the Arzela-Ascoli Theorem as used in $[\mathbf{1 0}]$ with slight modifications.

Proof. (i) Suppose $c$ is an essential non-peripheral simple closed curve in the geometric interior of $M$, parameterized on $[0,1]$ with constant speed. Let the length of $c$ be $|c|>0$. Then for each cusp $C_{i}$, there is an embedded neighborhood $N\left(C_{i}\right)$ of $C_{i}$ on $M$, bounded by a horocycle, such that each non-peripheral simple closed curve $c^{\prime}$ in the geometric interior of $M$ with length $\leq|c|$ cannot enter $N\left(C_{i}\right)$; for otherwise $c^{\prime}$ would be either peripheral or of infinite length. Now, let $M_{0}$ be $M$ with all the chosen horocycle neighborhoods $N\left(C_{i}\right)$ removed. Then $M_{0}$ is a compact metric subspace of $M$ with the induced hyperbolic metric. 
Now, choose a sequence of simple closed curves $\left\{c_{k}\right\}_{1}^{\infty}$, where each $c_{k}$ is parameterized on $[0,1]$ with constant speed, in the free homotopy class of $c$ (where the homotopy takes place in the geometric interior of $M$ ) such that their lengths $\leq|c|$ and are decreasing with limit the infimum of the lengths of the simple closed curves in the free homotopy class of $c$. Then by the Arzela-Ascoli Theorem (c.f. [10] Theorem A.19, p. 429), there is a subsequence of $\left\{c_{k}\right\}_{1}^{\infty}$, assumed to be $\left\{c_{k}\right\}_{1}^{\infty}$ itself, such that it converges uniformly to a closed curve $\gamma$ in $M_{0}$. It is clear that $\gamma$ is a geodesic since it is locally minimizing. Note that $\gamma$ is away from cusps by the choice of $\left\{c_{k}\right\}_{1}^{\infty}$. We claim that $\gamma$ cannot pass through any cone point. Suppose not, so that $\gamma$ passes through a cone point $P$. Then, for sufficiently large $k, c_{k}$ can be modified in the free homotopy class of $c$ to have length smaller than $|\gamma|$ (since the cone point has cone angle smaller than $\pi$ ), which is a contradiction. Thus, $\gamma$ must be a closed geodesic in the geometric interior of $M$. The uniqueness and simplicity of $\gamma$ can be proved by an easy argument since there are no bi-gons in the hyperbolic plane.

(ii) For an essential simple $\operatorname{arc} c$ in the geometric interior of $M$ which connects geometric boundary components, the proof of case (i) applies without modifications when none of the geometric boundary components involved is a cusp. Now, suppose at least one of the geometric boundary components involved is a cusp. For concreteness, let us assume that $c$ connects cusps $C_{1}$ to $C_{2}$. Remove suitable horocycle neighborhoods $N\left(C_{1}\right)$ and $N\left(C_{2}\right)$ respectively for $C_{1}$ and $C_{2}$ where the two horocycles are $H_{1}$ and $H_{2}$ respectively. Choose a simple arc $c_{0}$ in $M-N\left(C_{1}\right) \cup N\left(C_{2}\right)$ which goes along $c$ and connects $H_{1}$ to $H_{2}$. Let the length of $c_{0}$ be $\left|c_{0}\right|>0$. Now, for all other cusps $C_{i}$, there is a horocycle neighborhood $N\left(C_{i}\right)$ of $C_{i}$ on $M$ such that each non-peripheral simple closed curve $c^{\prime}$ in the geometric interior of $M$ with length $\leq\left|c_{0}\right|$ cannot enter $N\left(C_{i}\right)$. Again, let $M_{0}$ be $M$ with all the chosen horocycle neighborhoods $N\left(C_{i}\right)$ removed. By the same argument as in (i), we have a shortest simple geodesic realization $\gamma_{0}$ in the free relative homotopy class of $c_{0}$ in $M_{0}$ and $c_{0}$ does not pass through any cone point. Hence, $\gamma_{0}$ must be perpendicular to both $H_{1}$ and $H_{2}$ at its endpoints. Thus, $\gamma_{0}$ can be extended to a geodesic arc connecting $C_{1}$ to $C_{2}$. Again simplicity and uniqueness can be proved easily.

q.e.d.

The addendum can be verified easily since the realizations as degenerate simple geodesics in the respective cases are already known.

Remark 5.3. We make a remark that the following fact, whose proof is easy and hence omitted, is implicitly used throughout this paper: On a hyperbolic cone-surface for each cone point $P$ with angle less than $\pi$, there is a cone region $N(P)$, bounded by a suitable circle centered at $P$, such that if a geodesic $\gamma$ goes into $N(P)$, then either $\gamma$ will go directly 
to the cone point $P$ (hence, perpendicular to all the circles centered at $P)$ or $\gamma$ will develop a self-intersection in $N(P)$. The analogous fact for a cusp is used in [6], [14] and [19].

\section{Gaps between simple-normal $\Delta_{0}$-geodesics}

In this section we analyze the set of (oriented) geodesic rays emanating (perpendicularly) from $\Delta_{0}$. To illustrate the ideas, let us first consider the case where $\Delta_{0}$ is a boundary geodesic (the other two cases are similar); then the rays in this set can be identified naturally with the points on $\Delta_{0}$ itself. The set of complete, simple rays are the geodesic rays that develop fully (does not end in a boundary component), and are simple (no self-intersection); they form a Cantor set, as a subset of $\Delta_{0}$, by the analysis of [19], with Lebesgue measure zero by a generalization of the Birman-Series result (see Section 8). The complement, which is a countable collection of open intervals/gaps consists of rays which are either self-intersecting, or are simple and end at one of the boundary components (possibly $\Delta_{0}$ itself). There are also certain isolated geodesic rays in these gaps which are simple and terminate at a boundary component perpendicularly (in particular $\Delta_{0}$ itself), these will be useful for demarcating the gaps further, and for calculating the width of the gaps. It turns out to be simpler to analyze the set of rays which are either self-intersecting or terminate at a boundary component, rather than the complete simple rays, as done in [19]. In particular, we will not really need to know the Cantor set structure of the set of complete simple rays, only the Birman-Series result. We shall see that each such ray determines uniquely an embedded pair of pants in the cone surface where $\Delta_{0}$ is one of the boundary components of the pair of pants, such that the part of the geodesic ray up to the first point of self-intersection, or the intersection with a boundary component lies completely in the pair of pants. Furthermore, the width of the gap on which this geodesic ray lies on can be calculated from the lengths of the other two boundary components, together with the length of $\Delta_{0}$.

Let us fix throughout this section a compact hyperbolic cone-surface $M$ with a distinguished geometric boundary component $\Delta_{0}$. We first make some terminology for the classes of geodesics we are interested in.

Definition 6.1. A $\Delta_{0}$-geodesic on $M$ is an oriented geodesic ray which starts from $\Delta_{0}$ (and is perpendicular to it if $\Delta_{0}$ is a boundary geodesic) and is fully developed, that is, it develops forever, or until it terminates at a geometric boundary component. We denote by $\mathcal{G}\left(\Delta_{0}\right)$ (or just $\mathcal{G}$ ) the set of $\Delta_{0}$-geodesics.

A $\Delta_{0}$-geodesic is either non-simple or simple. It is regarded as nonsimple if and only if it intersects itself transversely at an interior point (a cone point is not treated as an interior point) or at a point on a 
boundary geodesic (this occurs only if $\Delta_{0}$ is a boundary geodesic and the point of intersection is on $\Delta_{0}$ ).

A simple $\Delta_{0}$-geodesic is either normal or not-normal in the following sense:

A simple $\Delta_{0}$-geodesic is normal if when fully developed either it never intersects any boundary geodesic or it intersects (hence terminates at) a boundary geodesic perpendicularly. Note that a simple-normal $\Delta_{0^{-}}$ geodesic may terminate at a cusp or a cone point. Thus, a simple $\Delta_{0^{-}}$ geodesic is not-normal if and only if it intersects a boundary geodesic (which might be $\Delta_{0}$ itself) obliquely.

We shall analyze the structure of all non-simple and simple-notnormal $\Delta_{0}$-geodesics and show that they form gaps between simplenormal $\Delta_{0}$-geodesics. Furthermore, the naturally measured widths of the suitably combined gaps are given by the Gap functions defined before in Section 4.

Note that McShane [19] analyzes directly all simple $\Delta_{0}$-geodesics (there are no simple-not-normal $\Delta_{0}$-geodesics in his case since there are no geodesic boundary components). Our analysis of the structure of $\Delta_{0}$-geodesics in the complement (non-simple and simple-not-normal) is a bit different from and actually simpler than that of McShane's. We shall show that these geodesics arise in the nice ways we expect, and are associated to canonical pairs of pants on the surface.

First, we parameterize all the $\Delta_{0}$-geodesics and define the widths for gaps between simple-normal $\Delta_{0}$-geodesics.

If $\Delta_{0}$ is a cusp, let $\mathcal{H}$ be a suitably chosen small horocycle as in McShane [19], see also [14]. If $\Delta_{0}$ is a cone point, let $\mathcal{H}$ be a suitably chosen small circle centered at $\Delta_{0}$. Let $\mathcal{H}$ be $\Delta_{0}$ itself if $\Delta_{0}$ is a boundary geodesic.

Then, each $\Delta_{0}$-geodesic has a unique first intersection point with $\mathcal{H}$, which is the starting point when $\Delta_{0}$ is a boundary geodesic. Note that the $\Delta_{0}$-geodesics intersect $\mathcal{H}$ orthogonally at their first intersection points. Thus, $\mathcal{G}$ can be naturally identified with $\mathcal{H}$, with the induced topology and measure. Let $\mathcal{H}_{\mathrm{ns}}, \mathcal{H}_{\mathrm{sn}}, \mathcal{H}_{\mathrm{snn}}$ be the point sets of the first intersections of $\mathcal{H}$ with respectively all non-simple, all simple-normal, all simple-not-normal $\Delta_{0}$-geodesics.

Proposition 6.2. The set $\mathcal{H}_{n s} \cup \mathcal{H}_{\text {snn }}$ is an open subset of $\mathcal{H}$ and hence $\mathcal{H}_{\text {sn }}$ is a closed subset of $\mathcal{H}$.

Proof. It is easy to see that the conditions of self-intersecting or ending obliquely at a boundary component is an open condition. q.e.d.

For the open subset $\mathcal{H}_{\mathrm{ns}} \cup \mathcal{H}_{\mathrm{snn}}$ of $\mathcal{H}$, we determine its structure by determining its maximal open intervals (which are the gaps we are looking for). By a generalized Birman-Series Theorem (see Section 8), the 
subset $\mathcal{H}_{\mathrm{sn}}$ of $\mathcal{H}$ has Hausdorff dimension 0 , and hence Lebesgue measure 0 . Therefore, the open subset $\mathcal{H}_{\mathrm{ns}} \cup \mathcal{H}_{\mathrm{snn}}$ of $\mathcal{H}$ has full measure, and our generalized McShane's identities (1.8)-(1.10) follow immediately.

Definition 6.3. A $\left[\Delta_{0}, \Delta_{0}\right]$-geodesic, $\gamma$, is an (oriented) $\Delta_{0}$-geodesic which terminates at $\Delta_{0}$ perpendicularly. (With the orientation, one can refer to its starting point and ending point.) Hence, the same geodesic with reversed orientation (hence with the starting and ending points interchanged) is also a $\left[\Delta_{0}, \Delta_{0}\right]$-geodesic, denoted by $-\gamma$.

We say that a $\left[\Delta_{0}, \Delta_{0}\right]$-geodesic $\gamma$ is a degenerate simple $\left[\Delta_{0}, \Delta_{0}\right]$ geodesic if $\Delta_{0}$ is not a $\pi$ cone point, and $\gamma$ is the double cover of a simple geodesic arc which connects $\Delta_{0}$ to an angle $\pi$ cone point, that is, $\gamma$ reaches the angle $\pi$ cone point along the simple geodesic arc and goes back to $\Delta_{0}$ along the same arc. Note that in this case $\gamma=-\gamma$.

We show that each non-degenerate simple $\left[\Delta_{0}, \Delta_{0}\right]$-geodesic $\gamma$ determines two maximal open intervals of $\mathcal{H}_{\mathrm{ns}} \cup \mathcal{H}_{\mathrm{snn}}$ as follows. (Their union is the main gap, defined later, determined by $\gamma$.)

Consider the configuration $\gamma \cup \mathcal{H}$. Assume $\gamma$ is non-degenerate and let $\mathcal{H}_{1}$ and $\mathcal{H}_{2}$ be the two sub-arcs with endpoints inclusive that $\gamma$ divides $\mathcal{H}$ into. Note that $\gamma$ intersects $\mathcal{H}$ twice (if $\mathcal{H}$ is taken to be a suitably small circle about $\Delta_{0}$ when $\Delta_{0}$ is a cone point). Let $\gamma_{0}$ be the sub-arc of $\gamma$ between the two intersection points. Thus, we have two simple closed curves $\mathcal{H}_{1} \cup \gamma_{0}$ and $\mathcal{H}_{2} \cup \gamma_{0}$ on $M$. Their geodesic realizations are disjoint generalized simple closed geodesics, denoted by $\alpha, \beta$ respectively (except when $M$ is a hyperbolic torus with a single geometric boundary component, in which case $\alpha=\beta)$. Note that $\alpha, \beta$ bound with $\Delta_{0}$ an embedded geometric pair of pants, denoted $\mathcal{P}(\gamma)$, on $M$.

Let $\delta_{\alpha}$ be the simple $\Delta_{0}$-geodesic arc in $\mathcal{P}(\gamma)$ which terminates at $\alpha$ and is normal to $\alpha$. Similarly, let $\delta_{\beta}$ be the simple $\Delta_{0}$-geodesic arc in $\mathcal{P}(\gamma)$ which terminates at $\beta$ and is normal to $\beta$. Let $[\alpha, \beta]$ be the simple geodesic arc in $\mathcal{P}(\gamma)$ which connects $\alpha$ and $\beta$ and is normal to them. See Figure 1.

Cutting $\mathcal{P}(\gamma)$ along $\delta_{\alpha}, \delta_{\beta}$ and $[\alpha, \beta]$, one obtains two pieces; let the one which contains the initial part of $\gamma$ be denoted by $\mathcal{P}^{+}(\gamma)$. There are two simple $\Delta_{0}$-geodesics, $\gamma_{\alpha}$ and $\gamma_{\beta}$, in $\mathcal{P}(\gamma)$ such that they are asymptotic to $\alpha$ and $\beta$ respectively, and such that their initial parts are contained in $\mathcal{P}^{+}(\gamma)$. See Figure 1 .

Lemma 6.4. Each $\Delta_{0}$-geodesic whose initial part lies in $\mathcal{P}^{+}(\gamma)$ between $\gamma_{\alpha}$ and $\gamma$ or between $\gamma$ and $\gamma_{\beta}$ is non-simple or simple-not-normal.

The union of these two gaps between simple-normal $\Delta_{0}$-geodesics formed by non-simple and simple-not-normal $\Delta_{0}$-geodesics is called the main gap determined by $\gamma$.

This lemma can be proved easily using a suitable model of the hyperbolic plane. The idea is that a $\Delta_{0}$-geodesic ray whose initial part 

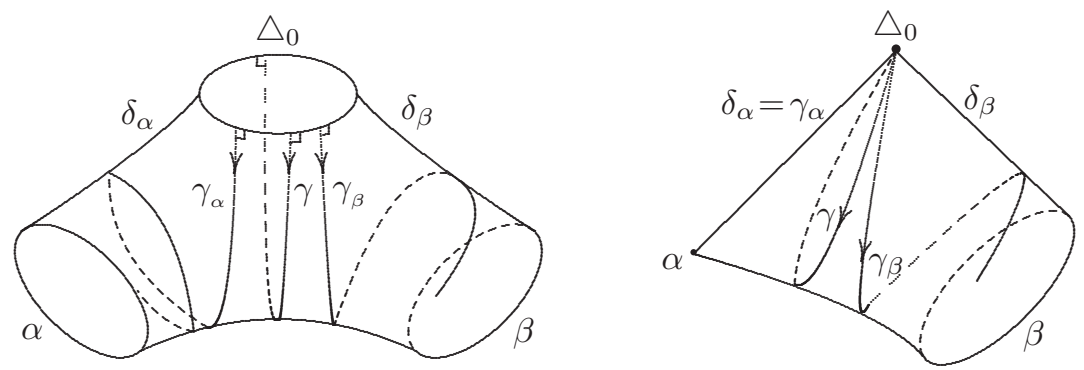

Figure 1.

lies in $\mathcal{P}^{+}(\gamma)$ between $\gamma_{\alpha}$ and $\gamma$ will not intersect $\gamma_{\alpha}$ or $\gamma$ directly, so it must come back to intersect for the first time either itself or $\Delta_{0}$, hence is either non-simple or simple, but not-normal (that is, intersecting $\Delta_{0}$ obliquely). More precisely, if $\Delta_{0}$ is a cusp or a cone point, all the $\Delta_{0}$-geodesics in the lemma are non-simple, while if $\Delta_{0}$ is a boundary geodesic, then there is a (critical) $\Delta_{0}$-geodesic, $\rho_{\gamma}$, whose initial part lies in $\mathcal{P}^{+}(\gamma)$ between $\gamma_{\alpha}$ and $\gamma$ such that $\rho_{\gamma}$ is non-simple and its only self-intersection is at its starting point on $\Delta_{0}$ (and hence terminates there) and it has the property that each $\Delta_{0}$-geodesic whose initial part lies in $\mathcal{P}^{+}(\gamma)$ between $\gamma_{\alpha}$ and $\rho_{\gamma}$ is non-simple, while each $\Delta_{0}$-geodesic whose initial part lies in $\mathcal{P}^{+}(\gamma)$ between $\rho_{\gamma}$ and $\gamma$ is simple-not-normal terminating at $\Delta_{0}$. There is a similar dichotomy for the $\Delta_{0}$-geodesics whose initial parts lie in $\mathcal{P}^{+}(\gamma)$ between $\gamma$ and $\gamma_{\beta}$.

Now, suppose one of $\alpha, \beta$, say $\alpha$, is a boundary geodesic. Then, there are two simple $\Delta_{0}$-geodesics in $\mathcal{P}(\gamma)$ which are asymptotes to $\alpha$. They are $\gamma_{\alpha}$ and $(-\gamma)_{\alpha}$.

The following lemma tells us that there is an extra gap determined by $\gamma$ in $\mathcal{P}^{+}(\gamma)$ between simple-normal $\Delta_{0}$-geodesics formed by simplenot-normal $\Delta_{0}$-geodesics.

Lemma 6.5. Each $\Delta_{0}$-geodesic whose initial part lies in $\mathcal{P}^{+}(\gamma)$ between $\delta_{\alpha}$ and $\gamma_{\alpha}$ is simple-not-normal.

This is almost self-evident from the geometry of the pair of pants $P(\gamma)$, and the proof is similar to that of the previous lemma.

Note that there is a similar and symmetric picture for the $\Delta_{0}$-geodesics whose initial parts lie in $\mathcal{P}^{-}(\gamma)$.

Hence, (for non-degenerate $\gamma$ ) in the geometric pair of pants $\mathcal{P}(\gamma)$, which is the same as $\mathcal{P}(-\gamma)$, if none of $\alpha, \beta$ is a boundary geodesic, then there are two main gaps determined by $\gamma$ and $-\gamma$ respectively; if (exactly) one of $\alpha, \beta$ is a boundary geodesic, then there are two extra gaps determined by $\gamma$ and $-\gamma$. 
The case of a degenerate simple $\left[\Delta_{0}, \Delta_{0}\right]$-geodesic $\gamma$ is handled in a similar way. Recall that $\gamma$ is the double cover of a $\Delta_{0}$-geodesic arc $\delta$ from $\Delta_{0}$ to an angle $\pi$ cone point $\alpha$. Then, there is a simple closed curve $\beta^{\prime}$, which is the boundary of a suitable regular neighborhood of $\Delta_{0} \cup \delta$ on $M$, such that $\beta^{\prime}$ bounds with $\Delta_{0}$ and $\alpha$ an embedded (topological) pair of pants. If $\Delta_{0}$ is not itself an angle $\pi$ cone point, then $\beta^{\prime}$ can be realized as an interior generalized simple closed geodesic $\beta$ which bounds with $\Delta_{0}$ and $\alpha$ an embedded pair of pants $\mathcal{H}\left(\Delta_{0}, \alpha, \beta\right)$ on $M$ and we can carry out the analysis as above with suitable modifications. In this case, $\gamma$ determines no gaps if $\Delta_{0}$ is itself an angle $\pi$ cone point. If $\Delta_{0}$ is not itself an angle $\pi$ cone point, then there are two main gaps, between $\gamma$ and each of the two $\Delta_{0}$-geodesics which are asymptotic to $\beta$ in $\mathcal{H}\left(\Delta_{0}, \alpha, \beta\right)$. We say that one of the two main gaps is determined by $\gamma$ and the other by $-\gamma$ although $\gamma=-\gamma$ in this case.

Definition 6.6. The width of an open subinterval $\mathcal{H}^{\prime}$ of $\mathcal{H}$ is defined respectively as:

(i) $\Delta_{0}$ is a cusp: the normalized parabolic measure, that is, the ratio of the Euclidean length of $\mathcal{H}^{\prime}$ to the Euclidean length of $\mathcal{H}$;

(ii) $\Delta_{0}$ is a cone point: the elliptic measure, that is, the angle (measured in radians) that $\mathcal{H}^{\prime}$ subtends with respect to the cone point $\Delta_{0}$

(iii) $\Delta_{0}$ is a boundary geodesic: the hyperbolic measure, that is, the hyperbolic length of $\mathcal{H}^{\prime}$ (recall that in this case, $\mathcal{H}$ is the same as the distinguished boundary geodesic $\Delta_{0}$ ).

Definition 6.7. The combined gap between simple-normal $\Delta_{0^{-}}$ geodesics determined by $\gamma$ is the union of the main gap and the extra gap (if there is any) determined by $\gamma$. The gap function $\operatorname{Gap}\left(\Delta_{0} ; \alpha, \beta\right)$ when $\Delta_{0}$ is a cone point or boundary geodesic or the normalized gap function $\operatorname{Gap}^{\prime}\left(\Delta_{0} ; \alpha, \beta\right)$ when $\Delta_{0}$ is a cusp is defined as the total width of the combined gap determined by $\gamma$, which is by symmetry the same as the total width of the combined gap determined by $-\gamma$.

We shall calculate the gap functions in Section 7.

On the other hand, the following key lemma shows that the nonsimple and simple-not-normal $\Delta_{0}$-geodesics obtained above are all the non-simple and simple-not-normal $\Delta_{0}$-geodesics.

Lemma 6.8. Each non-simple or simple-not-normal $\Delta_{0}$-geodesic lies in a main gap or an extra gap determined by some $\left[\Delta_{0}, \Delta_{0}\right]$-geodesic $\gamma$.

Proof. First, let $\delta$ be a non-simple $\Delta_{0}$-geodesic, with its first selfintersection point $Q$, where $Q$ lies in the geometric interior of $M$ or in $\Delta_{0}$ when $\Delta_{0}$ is a boundary geodesic. Let $\delta_{1}$ be the part of $\delta$ from starting point to $Q$; note that $\delta_{1}$ has the shape of a lasso. Then in the boundary of a suitable regular neighborhood of $\delta_{1}$, there is a simple 

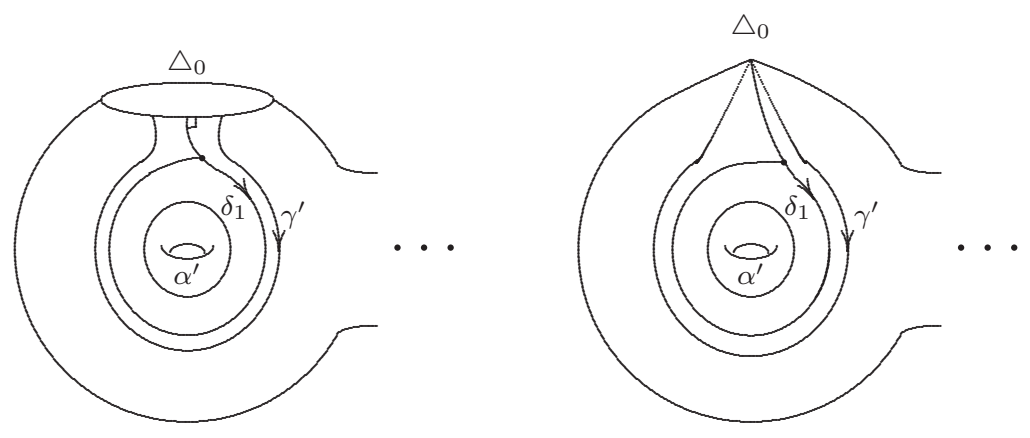

Figure 2.
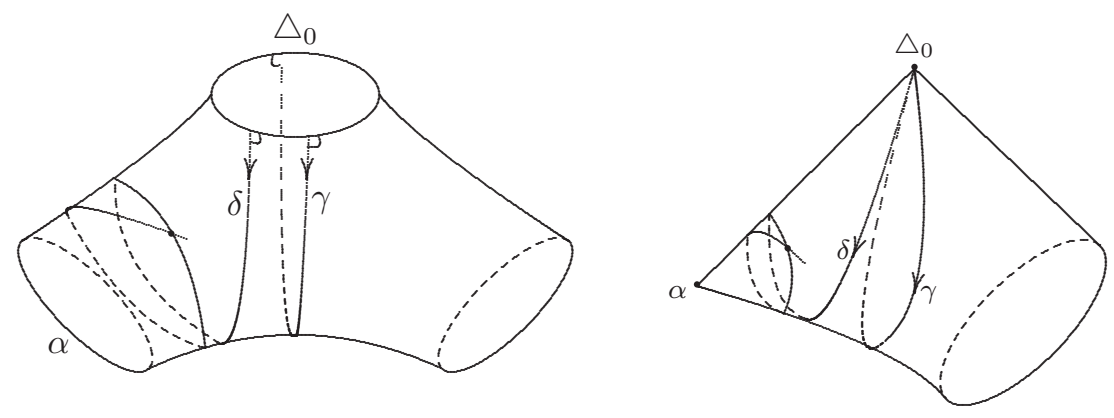

Figure 3.

arc $\gamma^{\prime}$ which connects $\Delta_{0}$ to itself and is disjoint from $\delta_{1}$ (except at $\Delta_{0}$ when $\Delta_{0}$ is a cone point); there is also a simple closed curve $\alpha^{\prime}$ which is freely homotopic to the loop part of $\delta_{1}$. See Figure 2. Let $\gamma, \alpha$ be the generalized simple closed geodesics on $M$ which realize $\gamma^{\prime}, \alpha^{\prime}$ in their respective free (relative) homotopy classes in the geometric interior of $M$. An easy geometric argument shows that $\alpha$ is disjoint from $\delta_{1}$ and that $\gamma$ is also disjoint from $\delta_{1}$ except at $\Delta_{0}$ when $\Delta_{0}$ is a cone point or a cusp. Furthermore, $\gamma$ and $\alpha$ cobound (together with $\Delta_{0}$ when $\Delta_{0}$ is a boundary geodesic) an embedded cylinder which contains $\delta_{1}$. Hence, the point in $\mathcal{H}$ which corresponds to the $\Delta_{0}$-geodesic $\delta$ lies in the main gap determined by $\gamma$. See Figure 3 .

Next, let $\delta$ be a simple-not-normal $\Delta_{0}$-geodesic which terminates at $\Delta_{0}$ itself; in this case, $\Delta_{0}$ is a boundary geodesic and $\mathcal{H}$ is $\Delta_{0}$ itself. Then, the boundary of a suitably chosen regular neighborhood of $\delta \cup \mathcal{H}$ consist of two disjoint simple closed curves in the geometric interior of $M$. Let their geodesic realizations be (disjoint) generalized simple closed geodesics $\alpha$ and $\beta$. Then, $\alpha, \beta$ bound with $\Delta_{0}$ an embedded pair of pants 
which contains $\delta$ in a main gap determined by the $\left[\Delta_{0}, \Delta_{0}\right]$-geodesic $\gamma$ which is the geodesic realization of $\delta$ in its free relative homotopy class.

Finally, let $\delta$ be a simple-not-normal $\Delta_{0}$-geodesic which terminates at a boundary geodesic $\Delta_{1}$ which is different from $\Delta_{0}$. The boundary of suitably chosen regular neighborhood of $\delta \cup \Delta_{1}$ on $M$ is a simple arc connecting $\Delta_{0}$ to itself and is disjoint from $\delta$. Its geodesic realization is a $\left[\Delta_{0}, \Delta_{0}\right]$-geodesic, $\gamma$, which is disjoint from $\delta$. Now, $\Delta_{1}, \gamma$ bound with $\Delta_{0}$ an embedded cylinder which contains $\delta$. Hence, $\delta$ lies in the extra gap determined by $\gamma$ or $-\gamma$.

q.e.d.

\section{Calculating the gap functions}

In this section, we calculate the gap function $\operatorname{Gap}\left(\Delta_{0} ; \alpha, \beta\right)$ when $\Delta_{0}$ is a cone point or a boundary geodesic, it is the width of the combined gap determined by a simple $\left[\Delta_{0}, \Delta_{0}\right]$-geodesic $\gamma$ on $M$.

Recall that $\alpha, \beta$ are the generalized simple closed geodesics determined by $\gamma$ and $\mathcal{P}(\gamma)$ is the geometric pair of pants that $\alpha, \beta$ bound with $\Delta_{0}$ on $M$.

Case 1. $\Delta_{0}$ is a cone point of cone angle $\theta \in(0, \pi]$.

In this case, the width of the main gap determined by $\gamma$ is the angle between $\gamma_{\alpha}$ and $\gamma_{\beta}$.

Let $x$ be the angle between $\delta_{\alpha}$ and $\gamma_{\alpha}$ and let $y$ be the angle between $\delta_{\beta}$ and $\gamma_{\beta}$.

Subcase 1.1. Both $\alpha$ and $\beta$ are interior generalized simple closed curves.

In this case, the width of the combined gap determined by $\gamma$ is the angle between $\gamma_{\alpha}$ and $\gamma_{\beta}$ and is equal to $\frac{\theta}{2}-(x+y)$.

By a formula in Fenchel [12] VI.3.2 (line 10, page 87),

$$
\begin{aligned}
& \sinh \left|\delta_{\alpha}\right|=\frac{\cosh \frac{|\beta|}{2}+\cos \frac{\theta}{2} \cosh \frac{|\alpha|}{2}}{\sin \frac{\theta}{2} \sinh \frac{|\alpha|}{2}}, \\
& \sinh \left|\delta_{\beta}\right|=\frac{\cosh \frac{|\alpha|}{2}+\cos \frac{\theta}{2} \cosh \frac{|\beta|}{2}}{\sin \frac{\theta}{2} \sinh \frac{|\beta|}{2}} .
\end{aligned}
$$

Hence

$$
\begin{aligned}
& \tan x=\frac{1}{\sinh \left|\delta_{\alpha}\right|}=\frac{\sin \frac{\theta}{2} \sinh \frac{|\alpha|}{2}}{\cosh \frac{|\beta|}{2}+\cos \frac{\theta}{2} \cosh \frac{|\alpha|}{2}}, \\
& \tan y=\frac{1}{\sinh \left|\delta_{\beta}\right|}=\frac{\sin \frac{\theta}{2} \sinh \frac{|\beta|}{2}}{\cosh \frac{|\alpha|}{2}+\cos \frac{\theta}{2} \cosh \frac{|\beta|}{2}} .
\end{aligned}
$$




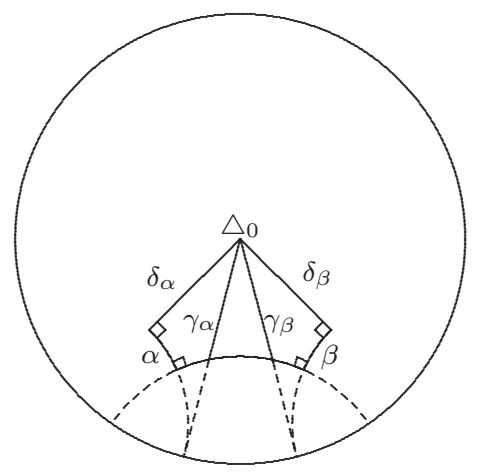

Figure 4. Subcase 1.1.

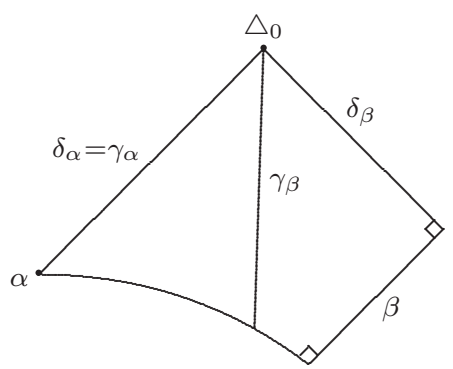

Figure 5. Subcase 1.3.

From these, one can derive that

$$
\tan (x+y)=\frac{\sin \frac{\theta}{2} \sinh \frac{|\alpha|+|\beta|}{2}}{1+\cos \frac{\theta}{2} \cosh \frac{|\alpha|+|\beta|}{2}}
$$

and hence that

$$
\tan \frac{x+y}{2}=\tan \frac{\theta}{4} \tanh \frac{|\alpha|+|\beta|}{4} .
$$

Thus

$$
\begin{aligned}
\tan \left(\frac{\theta}{4}-\frac{x+y}{2}\right) & =\frac{\tan \frac{\theta}{4}\left(1-\tanh \frac{|\alpha|+|\beta|}{4}\right)}{1+\tan ^{2} \frac{\theta}{4} \tanh \frac{|\alpha|+|\beta|}{4}} \\
& =\frac{\sin \frac{\theta}{2}}{\cos \frac{\theta}{2}+\exp \frac{|\alpha|+|\beta|}{2}} .
\end{aligned}
$$

Hence, in this case, we have

$$
\begin{aligned}
\operatorname{Gap}\left(\Delta_{0} ; \alpha, \beta\right) & =\frac{\theta}{2}-(x+y) \\
& =2 \tan ^{-1}\left(\frac{\sin \frac{\theta}{2}}{\cos \frac{\theta}{2}+\exp \frac{|\alpha|+|\beta|}{2}}\right) .
\end{aligned}
$$

Subcase 1.2. $\alpha$ is a boundary geodesic and $\beta$ is an interior generalized simple closed geodesic.

In this case, the width of the combined gap determined by $\gamma$ is the angle between $\delta_{\alpha}$ and $\gamma_{\beta}$ and is equal to $\frac{\theta}{2}-y$. Hence, by (7.4), we have

$$
\operatorname{Gap}\left(\Delta_{0} ; \alpha, \beta\right)=\frac{\theta}{2}-\tan ^{-1}\left(\frac{\sin \frac{\theta}{2} \sinh \frac{|\beta|}{2}}{\cosh \frac{|\alpha|}{2}+\cos \frac{\theta}{2} \cosh \frac{|\beta|}{2}}\right) .
$$


Subcase 1.3. $\alpha$ is a cone point of cone angle $\varphi \in(0, \pi]$ and $\beta$ is an interior generalized simple closed geodesic.

Note that, in this case, $\gamma_{\alpha}$ coincides with $\delta_{\alpha}$ and hence $x=0$. Therefore, the width of the combined gap determined by $\gamma$ is the angle between $\delta_{\alpha}$ and $\gamma_{\beta}$ and is equal to $\frac{\theta}{2}-y$.

Now, by a formula in Fenchel [12] VI.3.3 (line 13, p. 88),

$$
\sinh \left|\delta_{\beta}\right|=\frac{\cos \frac{\varphi}{2}+\cos \frac{\theta}{2} \cosh \frac{|\beta|}{2}}{\sin \frac{\theta}{2} \sinh \frac{|\beta|}{2}} .
$$

Hence

$$
\tan y=\frac{1}{\sinh \left|\delta_{\beta}\right|}=\frac{\sin \frac{\theta}{2} \sinh \frac{|\beta|}{2}}{\cos \frac{\varphi}{2}+\cos \frac{\theta}{2} \cosh \frac{|\beta|}{2}} .
$$

Thus, in this case, we have

$$
\operatorname{Gap}\left(\Delta_{0} ; \alpha, \beta\right)=\frac{\theta}{2}-\tan ^{-1}\left(\frac{\sin \frac{\theta}{2} \sinh \frac{|\beta|}{2}}{\cos \frac{\varphi}{2}+\cos \frac{\theta}{2} \cosh \frac{|\beta|}{2}}\right) .
$$

Case 2. $\Delta_{0}$ is a boundary geodesic of length $l>0$.

In this case, the width of the main gap determined by $\gamma$ is the distance between $\gamma_{\alpha}$ and $\gamma_{\beta}$ along $\Delta_{0}$.

Let $x$ be the distance between $\delta_{\alpha}$ and $\gamma_{\alpha}$ along $\Delta_{0}$ and let $y$ be the distance between $\delta_{\beta}$ and $\gamma_{\beta}$ along $\Delta_{0}$.

We shall see that all calculations in this case are parallel to those in Case 1.

Subcase 2.1. Both $\alpha$ and $\beta$ are interior generalized simple closed curves.

In this case, the width of the combined gap determined by $\gamma$ is the distance between $\gamma_{\alpha}$ and $\gamma_{\beta}$ along $\Delta_{0}$ and is equal to $\frac{l}{2}-(x+y)$.

By the cosine rule for right angled hexagons on the hyperbolic plane (c.f. Fenchel [12] VI.3.1, page 86, or Beardon [4] Theorem 7.19.2, page 161),

$$
\begin{aligned}
& \cosh \left|\delta_{\alpha}\right|=\frac{\cosh \frac{|\beta|}{2}+\cosh \frac{l}{2} \cosh \frac{|\alpha|}{2}}{\cosh \frac{l}{2} \sinh \frac{|\alpha|}{2}}, \\
& \cosh \left|\delta_{\beta}\right|=\frac{\cosh \frac{|\alpha|}{2}+\cosh \frac{l}{2} \cosh \frac{|\beta|}{2}}{\sinh \frac{l}{2} \sinh \frac{|\beta|}{2}} .
\end{aligned}
$$

Hence

$$
\tanh x=\frac{1}{\cosh \left|\delta_{\alpha}\right|}=\frac{\sinh \frac{l}{2} \sinh \frac{|\alpha|}{2}}{\cosh \frac{|\beta|}{2}+\cosh \frac{l}{2} \cosh \frac{|\alpha|}{2}},
$$




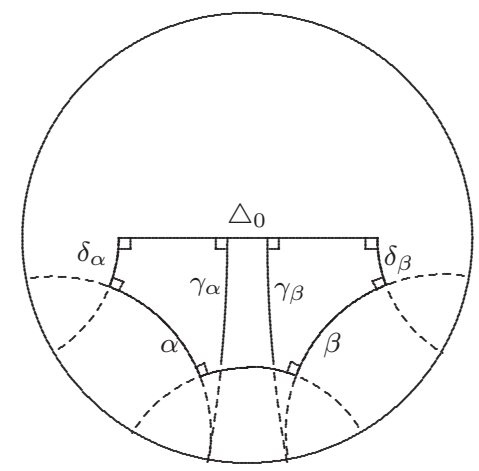

Figure 6. Subcase 2.1.

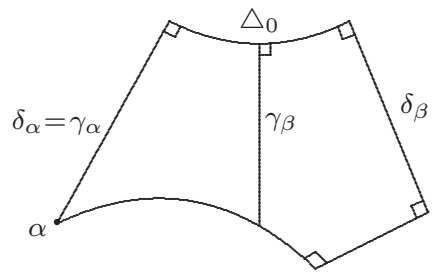

Figure 7. Subcase 2.3.

$$
\tanh y=\frac{1}{\cosh \left|\delta_{\beta}\right|}=\frac{\sinh \frac{l}{2} \sinh \frac{|\beta|}{2}}{\cosh \frac{|\alpha|}{2}+\cosh \frac{l}{2} \cosh \frac{|\beta|}{2}} .
$$

From these, one can derive that

$$
\tanh (x+y)=\frac{\sinh \frac{l}{2} \sinh \frac{|\alpha|+|\beta|}{2}}{1+\cosh \frac{l}{2} \cosh \frac{|\alpha|+|\beta|}{2}}
$$

and hence that

$$
\tanh \frac{x+y}{2}=\tanh \frac{l}{4} \tanh \frac{|\alpha|+|\beta|}{4} .
$$

Thus,

$$
\begin{aligned}
\tanh \left(\frac{l}{4}-\frac{x+y}{2}\right) & =\frac{\tanh \frac{l}{4}\left(1-\tanh \frac{|\alpha|+|\beta|}{4}\right)}{1-\tanh ^{2} \frac{l}{4} \tanh \frac{|\alpha|+|\beta|}{4}} \\
& =\frac{\sinh \frac{l}{2}}{\cosh \frac{l}{2}+\exp \frac{|\alpha|+|\beta|}{2}} .
\end{aligned}
$$

Hence, in this case, we have

$$
\begin{aligned}
\operatorname{Gap}\left(\Delta_{0} ; \alpha, \beta\right) & =\frac{l}{2}-(x+y) \\
& =2 \tanh ^{-1}\left(\frac{\sinh \frac{l}{2}}{\cosh \frac{l}{2}+\exp \frac{|\alpha|+|\beta|}{2}}\right) .
\end{aligned}
$$

Subcase 2.2. $\alpha$ is a boundary geodesic and $\beta$ is an interior generalized simple closed geodesic.

In this case, the width of the combined gap determined by $\gamma$ is the distance between $\delta_{\alpha}$ and $\gamma_{\beta}$ along $\Delta_{0}$ and is equal to $\frac{l}{2}-y$. Hence, by 
(7.16), we have

$$
\operatorname{Gap}\left(\Delta_{0} ; \alpha, \beta\right)=\frac{l}{2}-\tanh ^{-1}\left(\frac{\sinh \frac{l}{2} \sinh \frac{|\beta|}{2}}{\cosh \frac{|\alpha|}{2}+\cosh \frac{l}{2} \cosh \frac{|\beta|}{2}}\right) .
$$

Subcase 2.3. $\alpha$ is a cone point of cone angle $\varphi \in(0, \pi]$ and $\beta$ is an interior generalized simple closed geodesic.

Note that, in this case, $\gamma_{\alpha}$ coincides with $\delta_{\alpha}$ and hence $x=0$. Hence, the width of the combined gap determined by $\gamma$ is the distance between $\delta_{\alpha}$ and $\gamma_{\beta}$ along $\Delta_{0}$ and is equal to $\frac{l}{2}-y$.

Now, by a formula in Fenchel [12] VI.3.2 (line 8, p. 87),

$$
\cosh \left|\delta_{\beta}\right|=\frac{\cos \frac{\varphi}{2}+\cosh \frac{l}{2} \cosh \frac{|\beta|}{2}}{\sinh \frac{l}{2} \sinh \frac{|\beta|}{2}} .
$$

Hence

$$
\tanh y=\frac{1}{\cosh \left|\delta_{\beta}\right|}=\frac{\sinh \frac{l}{2} \sinh \frac{|\beta|}{2}}{\cos \frac{\varphi}{2}+\cosh \frac{l}{2} \cosh \frac{|\beta|}{2}} .
$$

Thus, in this case, we have

$$
\operatorname{Gap}\left(\Delta_{0} ; \alpha, \beta\right)=\frac{l}{2}-\tanh ^{-1}\left(\frac{\sinh \frac{l}{2} \sinh \frac{|\beta|}{2}}{\cos \frac{\varphi}{2}+\cosh \frac{l}{2} \cosh \frac{|\beta|}{2}}\right) .
$$

Remark 7.1. We remark that the formulae in Case 0 for the normalized width $\operatorname{Gap}^{\prime}\left(\Delta_{0} ; \alpha, \beta\right)$ when $\Delta_{0}$ is a cusp can be derived by similar (and simpler) calculations or by considering the first order infinitesimal terms of those formulae with respect to $\theta$ in Case 1 or with respect to $l$ in Case 2. Hence, all derivations in Case 0 are omitted.

\section{Generalization of the Birman-Series Theorem}

The celebrated Birman-Series Theorem [6] in its simplest form states that complete simple geodesics on a closed hyperbolic surface are sparsely distributed.

A simple extension of the techniques used to prove the above shows that for a hyperbolic surface $M$ with cusps, the set of simple geodesic rays emanating from a distinguished cusp $\Delta_{0}$ has Lebesgue measure 0; that is, if we identify the set of all geodesic rays emanating from the cusp $\Delta_{0}$ with a small horocycle $\mathcal{H}$ around $\Delta_{0}$ as in Section 6 , with the standard horocyclic measure on $\mathcal{H}$, then the subset of $\mathcal{H}$ corresponding to the simple geodesic rays has Lebesgue measure 0 . This was the result used by McShane in [19], to show that the sum of the gaps indeed gave the full measure. The aim of this section is to show that the same result holds for a hyperbolic cone surface $M$ with geometric boundary where 
each cone point has cone angle in $(0, \pi]$. The techniques are similar to [6], we will omit details.

For clarity of exposition, let us assume that $\Delta_{0}$ is a cone point, and that there are no geodesic boundary components, so all boundary components are either cone points or cusps. It is not difficult to see that the arguments below extend to when there are geodesic boundary components as well (for example, by a doubling argument), or when $\Delta_{0}$ is a cusp or boundary geodesic. We are interested in the set $\mathcal{G}$ of geodesic rays emanating from $\Delta_{0}$, which can be identified with a suitably chosen small circle $\mathcal{H}$ about $\Delta_{0}$, as in Section 6 , with the standard measure. We wish to show that the subset of $\mathcal{H}$ corresponding to the simple normal geodesic rays has Lebesgue measure zero. It suffices to consider the non-terminating simple geodesic rays (those that do not end at a geometric boundary) since the terminating ones are countable, and in fact are the boundary of gaps on both sides and so can be absorbed into the calculation of the gap measures.

We start by cutting $M$ along normal geodesics connecting cusps or cone points to form a (convex, by the cone angle conditions) fundamental polygon for $M$ in the hyperbolic plane. All vertices of the polygon correspond to either cusps or cone points of $M$. We now truncate the polygon by replacing each vertex corresponding to a cusp by a suitably small horocycle; all geodesics on $M$ which intersect this horocycle will either go directly into the cusp or be self intersecting, see Remark 5.3. Call the resulting convex polygon $R$, this will now have finite diameter, and will be a fundamental polygon for $\bar{M}$, which is $M$ with some horocyclic neighborhood of the cusps removed. All non-terminating, simple geodesic rays from $\Delta_{0}$ are contained in $\bar{M}$. Let $A=\left\{a_{1}, a_{2}, \ldots, a_{m}\right\}$ denote the ordered set of vertices and oriented sides of $R$ with anticlockwise ordering, fixing $a_{1}$ so that it corresponds to $\Delta_{0}$. Then for odd $i, a_{i}$ are vertices of $R$, for even $i, a_{i}$ are (oriented) sides of $R$, and there is a side-pairing pattern for $R$.

A simple non-terminating geodesic ray emanating from $\Delta_{0}$ intersects the projection of $\partial R$ onto $\bar{M}$ in an infinite sequence of partition points $t_{0}, t_{1}, \ldots, t_{n}, \ldots$ on the geodesic, where $t_{0}$ is the initial point of the ray, and so on. This gives rise to an infinite sequence of non-intersecting geodesic arcs on $R$, with the endpoints of each geodesic arc lying on $\partial R$, hence, each arc corresponds to a pair of elements in $A$. Each partition point $t_{i}$ with $i \geq 1$ corresponds to the end point of one arc and the starting point of the next, denote by $a\left(t_{i}\right)$ the element of $A$ where the end point of the arc corresponding to $t_{i}$ lies, for example, $a\left(t_{1}\right)$ is the element of $A$ on which the endpoint of the first geodesic arc lies. Now, take the finite part of the ray, call it $\gamma$, which gives rise to just the first $n$ arcs of the sequence, we get what is known as a simple diagram of length $n$ on $R$ (see [6]), which is a collection of $n$ pairwise disjoint (geodesic) 


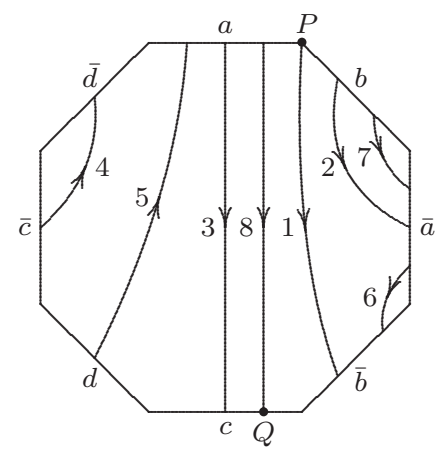

Figure 8. Simple diagram for a geodesic arc.

arcs joining pairs of distinct elements of $A$. Note that only the initial point of $\gamma$ lies on a vertex of $R$, all other end points of the arcs lie on sides of $R$. Two simple diagrams are regarded as being identical if they agree up to isotopy supported on each side of $R$. For $a_{i}, a_{j} \in A, i \neq j$, let $n_{i j}$ denote the number of arcs joining $a_{i}$ to $a_{j}$ in the given simple diagram, and let $h_{1}(\gamma)=\left(n_{12}, n_{13}, \ldots, n_{m-1, m}\right)$. Let $h_{2}(\gamma)$ record positions of the initial and final points of $\gamma$ in the simple diagram. See Figure 8 which shows the schematic picture of the simple diagram for a geodesic arc emanating from the cone point of a genus two surface with one cone point, where $P$ is the initial point and $Q$ the terminal point of the arc.

More generally, denote by $J_{0}(n)$ the set of oriented simple geodesic $\operatorname{arcs} \gamma$ on $\bar{M}$ such that the initial and final points of $\gamma$ lie on the projection of $\partial R$ to $\bar{M}$ and $\gamma$ gives rise to a simple diagram of length $n$ on $R$. We say that the combinatorial length of $\gamma$ (with respect to $R$ ) is $n$, and denote it by $\|\gamma\|$. Then, the following results of [6] still hold:

Lemma 8.1. Suppose that $\gamma, \gamma^{\prime} \in J_{0}$ and that $h_{1}(\gamma)=h_{1}\left(\gamma^{\prime}\right), h_{2}(\gamma)$ $=h_{2}\left(\gamma^{\prime}\right)$. Let $t_{0}, t_{1}, \ldots, t_{n}$ and $t_{0}^{\prime}, t_{1}^{\prime}, \ldots, t_{n}^{\prime}$ be the partition points of $\gamma, \gamma^{\prime}$ respectively. Then, $a\left(t_{i}\right)=a\left(t_{i}^{\prime}\right)$ for each $i=1,2, \ldots, n$.

Lemma 8.2. Let $J_{0}(n)=\left\{\gamma \in J_{0} \mid\|\gamma\|=n\right\}$. Then, there is a polynomial $P_{0}(\cdot)$ such that the number of simple diagrams of length $n$

$$
\operatorname{card}\left\{\left(h_{1}(\gamma), h_{2}(\gamma)\right) \mid \gamma \in J_{0}(n)\right\} \leq P_{0}(n) .
$$

The results above as applied to simple, non-terminating geodesic rays emanating from $\Delta_{0}$ says that first, the initial $n$ cutting sequence of the geodesic $\gamma$ with $\partial R$ is determined by $h_{1}(\gamma)$ and $h_{2}(\gamma)$, and secondly, there is a polynomial bound $P(n)$ on the number of possibilities for such cutting sequences. On the other hand, a simple geodesic segment with large combinatorial length $n$ is long. When all cone angles are less than $\pi$, this follows from the fact that each geodesic segment of the simple diagram is bounded below by some fixed constant $\alpha>0$, where $\alpha$ depends only on $R$, since segments connecting two non-adjacent sides 
are certainly bounded below by a positive constant, segments connecting adjacent sides are bounded by Remark 5.3. A slight refinement is needed if there are cone angles equal to $\pi$, see for example [6] for the arguments needed. Finally, two simple geodesic rays emanating from $\Delta_{0}$ with the same initial cutting sequence for large $n$ travel within a bounded distance of each other for a long time (here, we use the fact that the polygon $R$ has finite diameter), hence, start out exponentially close together. These last two results are formalized in the following lemmas.

Lemma 8.3. There is a universal constant $\alpha>0$ (depending only on the choice of the fundamental polygon $R$ ) so that

$$
l(\gamma) \geq \alpha\|\gamma\|
$$

for $\gamma \in J_{0}$ with $\|\gamma\|$ sufficiently large, where $l(\gamma)$ denotes the hyperbolic length of $\gamma$.

Lemma 8.4. Suppose $\gamma$ and $\gamma^{\prime}$ are simple, non-terminating geodesics emanating from $\Delta_{0}$ such that the first $n$ cutting sequence of $\gamma$ and $\gamma^{\prime}$ with $R$ are identical, that is, $a_{i}(\gamma)=a_{i}\left(\gamma^{\prime}\right)$ for $i=1, \ldots, n$. Let $p(\gamma)$ and $p\left(\gamma^{\prime}\right)$ be the points on $\mathcal{H}$ corresponding to $\gamma$ and $\gamma^{\prime}$ respectively, where $\mathcal{H}$ is a fixed circle of small radius about $\Delta_{0}$, equipped with the standard length measure || . Then, there exists universal constants $\alpha, c$ such that $\left|p\left(\gamma^{\prime}\right)-p(\gamma)\right|<c e^{-\alpha n}$.

From the above, it follows that the set of simple non-terminating geodesic rays emanating from a fixed cone point $\Delta_{0}$ regarded as a subset of $\mathcal{H}$ has an open cover by $\leq P(n)$ open intervals of length $<c e^{-\alpha n}$, letting $n \rightarrow \infty$, we see the Lebesgue measure is 0 . Slight modifications of the arguments above apply to the cases where $\Delta_{0}$ is a boundary geodesic or cusp.

Finally, we remark that the above Birman-Series' arguments will give rough estimates (upper bounds) for the number of simple closed geodesics with the same combinatorial length on a compact hyperbolic cone-surface $M$, which is enough for proving the absolute convergence of the series appearing in various generalized McShane's identities, as was observed and used in [2], (for the case of complete hyperbolic surfaces) for similar purposes.

Lemma 8.5. Let $M$ be a compact hyperbolic cone-surface with all cone angles in $(0, \pi]$. Then, for any constant $c>0$

(i) the series

$$
\sum_{\beta} \frac{1}{\exp (c|\beta|)}
$$

converges absolutely, where the sum is taken over all generalized simple closed geodesics on $M$ and all simple normal geodesic arcs connecting geometric boundary components of $M$; 
(ii) the series

$$
\sum_{\alpha, \beta} \frac{1}{\exp (c(|\alpha|+|\beta|))}
$$

converges absolutely, where the sum is taken over all pairs $\alpha, \beta$ of disjoint generalized simple closed geodesics on $M$ and/or simple normal geodesic arcs connecting geometric boundary components of $M$.

The idea of the proof is that, in the case of (i), each $\beta$ determines and is determined by a unique complete simple diagram which contains the complete information of $\beta$ (in the case where $\beta$ is a simple closed geodesic, the diagram is closed, that is, we have $t_{n}=t_{0}$ using earlier notation). Hence, the number of all such $\beta$ with combinatorial length $\|\beta\|=n$ is bounded by the polynomial value $P_{0}(n)$. Note that each $\beta$ has hyperbolic length $|\beta|$ at least $\kappa\|\beta\|$ for some constant $\kappa>0$ depending only on the chosen fundamental domain $R$; hence, we have $c|\beta| \geq c \kappa\|\beta\|$. It follows that

$$
\begin{aligned}
\sum_{\beta} \frac{1}{\exp (c|\beta|)} & \leq \sum_{n=1}^{\infty} \sum_{\|\beta\|=n} \frac{1}{\exp (c \kappa\|\beta\|)} \\
& \leq \sum_{n=1}^{\infty} \frac{P_{0}(n)}{\exp (c \kappa n)}
\end{aligned}
$$

and hence the series in (8.1) converges. One can prove (8.2) similarly since each pair $\alpha, \beta$ can be determined by a suitable complete simple diagram. The referee has also pointed out that the above upper bounds can be proven by a direct application of train tracks.

\section{Proof of Theorems}

Proof of Theorem 1.8. Now, the proof is obvious from the previous discussions. Suppose $\Delta_{0}$ is a cone point. Recall $\mathcal{H}$ is a suitably chosen small circle centered at $\Delta_{0}$, and $\mathcal{H}_{\mathrm{ns}}, \mathcal{H}_{\mathrm{sn}}, \mathcal{H}_{\mathrm{snn}}$ are the point sets of the first intersections of $\mathcal{H}$ with respectively all non-simple, all simplenormal, all simple-not-normal $\Delta_{0}$-geodesics. The elliptic measure of each of these subsets of $\mathcal{H}$ is the radian measure that it subtends to the cone point $\Delta_{0}$. The generalized Birman-Series Theorem in Section 8 implies that the closed subset $\mathcal{H}_{\mathrm{sn}}$ has measure 0 . Hence, the open subset $\mathcal{H}_{\mathrm{ns}} \cup \mathcal{H}_{\mathrm{snn}}$ has full measure, that is, $\theta_{0}$. Now, the maximal open intervals of $\mathcal{H}_{\mathrm{ns}} \cup \mathcal{H}_{\mathrm{snn}}$, suitably combined, have measure $2 \operatorname{Gap}\left(\Delta_{0} ; \alpha, \beta\right)$ for each unordered pair of generalized simple closed geodesics $\alpha, \beta$ on $M$ which bound with $\Delta_{0}$ an embedded pair of pants on $M$. Hence, their sum is equal to $\theta_{0}$ and the desired identity follows. The cases where $\Delta_{0}$ is a boundary geodesic or a cusp are similarly proved.

q.e.d. 
Proof of Corollary 1.10. Consider the case where $\Delta_{0}$ is a cone point. In this case, $T$ admits a unique elliptic involution $\eta$ such that $\eta$ maps each oriented simple closed geodesics on $T$ onto itself with orientation reversed. Note that $\eta$ fixes the cone point $\Delta_{0}$ and three other interior points which are the so-called Weierstrass points of $T$. Each simple closed geodesics on $T$ passes exactly two Weierstrass points; hence, there are three Weierstrass classes of simple closed geodesics on $T$. Now, the quotient of $T$ under $\eta$ is a sphere with three angle $\pi$ cone points and a cone point with angle $\theta / 2$. Then, Theorem 1.8 applies to $M=T /\langle\eta\rangle$, with $\Delta_{0}$ the angle $\pi$ cone point whose inverse image under $\eta$ is the Weierstrass point that the Weierstrass class $\mathcal{A}$ misses. Note that each generalized simple closed geodesic on $M=T /\langle\eta\rangle$ is either a geometric boundary component or degenerate simple closed geodesic which is the double cover of a simple geodesic arc which connects two Weierstrass points. Hence, the set of all pairs of generalized simple closed geodesics which bound with $\Delta_{0}$ an embedded pair of pants is exactly the set of pairs consisting of the angle $\theta / 2$ cone point plus a degenerate simple closed geodesic $\gamma^{\prime}$ which is the double cover of the quotient simple geodesic arc of a simple closed geodesic $\gamma$ on $T$ in the given Weierstrass class $\mathcal{A}$ (note that by definition the length of $\gamma^{\prime}$ is the same as that of $\gamma)$. Hence, by (4.7), the summand in the summation is

$$
\frac{\pi}{2}-\tan ^{-1}\left(\frac{\sin \frac{\pi}{2} \sinh \frac{|\gamma|}{2}}{\cos \frac{\theta}{4}+\cos \frac{\pi}{2} \cosh \frac{|\gamma|}{2}}\right)=\tan ^{-1}\left(\frac{\cos \frac{\theta}{4}}{\sinh \frac{|\gamma|}{2}}\right) .
$$

The proof for the case where $\Delta_{0}$ is a boundary geodesic is similar.

q.e.d.

Remark 9.1. Note that we can also choose $\Delta_{0}$ to be the angle $\theta / 2$ cone point on $T /\langle\eta\rangle$, then we obtain (1.4), the generalization of McShane's original identity to the cone-torus $T$. This is one way of seeing why we can allow the cone angle of up to $2 \pi$ in the cone torus case.

Proof of Theorem 1.13. It is well known that $M$ admits a unique hyperelliptic involution $\eta$ (see for example [15]) such that $\eta$ maps each simple closed geodesic onto itself and preserves/reverses the orientation of separating/non-separating simple closed geodesics. Note that $\eta$ leaves six points on $M$ fixed; they are the six Weierstrass points on $M$. Consider the quotient $M^{\prime}=M /\langle\eta\rangle$ which is a sphere with six angle $\pi$ cone points. Each generalized simple closed geodesic on $M^{\prime}$ is either

(i) an angle $\pi$ cone point; or

(ii) a degenerate simple closed geodesic $\beta^{\prime}$ which is the double cover of a simple geodesic arc $c$ connecting two angle $\pi$ cone points where the inverse image of $c$ under $\eta$ is a non-separating simple closed geodesic $\beta$ on $M$; or 
(iii) a separating (non-degenerate) simple closed geodesic $\alpha^{\prime}$ whose inverse image under $\eta$ is a separating simple closed geodesic $\alpha$ on $M$. In this case, $\alpha^{\prime}$ does not pass through any of the six angle $\pi$ cone points and there are three of them on each side of $\alpha^{\prime}$ on $M^{\prime}$. Hence, $\alpha$ passes none of six Weierstrass points and there are three of them on each side of $\alpha$ on $M$.

Now, apply Theorem 1.8 to $M^{\prime}$ with $\Delta_{0}$ one of the six angle $\pi$ cone points. Then, each pair of generalized simple closed geodesics on $M^{\prime}$ which bound with $\Delta_{0}$ an embedded pair of pants $\mathcal{P}$ consists of a separating simple closed geodesic $\alpha^{\prime}$ on $M^{\prime}$ and a degenerate simple closed geodesic $\beta^{\prime}$ on $M^{\prime}$ which lies on the same side of $\alpha^{\prime}$ as $\Delta_{0}$ and misses $\Delta_{0}$. Let the inverse image of $\alpha^{\prime}, \beta^{\prime}$ under $\eta$ be $\alpha, \beta$. Then, $\alpha$ is a separating simple closed geodesic on $M$ and $\beta$ is a non-separating simple closed geodesic on $M$. Furthermore, $\beta$ and the Weierstrass point which is the inverse image of $\Delta_{0}$ lie on the same side of $\alpha$ on $M$. Note that the hyperbolic lengths of $\alpha^{\prime}, \beta^{\prime}$ are respectively $|\alpha| / 2,|\beta|$. Hence by (4.5), in this case, the summand in the resulting generalized McShane's Weierstrass identity for $M^{\prime}$ with the chosen $\Delta_{0}$ is

$$
2 \tan ^{-1}\left(\frac{\sin \frac{\pi}{2}}{\cos \frac{\pi}{2}+\exp \frac{|\alpha| / 2+|\beta|}{2}}\right)=2 \tan ^{-1} \exp \left(-\frac{|\alpha|}{4}-\frac{|\beta|}{2}\right) .
$$

Note that each pair of disjoint simple closed geodesics $(\alpha, \beta)$ on $M$ such that $\alpha$ is separating and $\beta$ is non-separating arises as the inverse image of a unique pair of generalized simple closed geodesics on $M^{\prime}$ as described above, where the chosen $\Delta_{0}$ is the angle $\pi$ cone point which is the image under $\eta$ of the Weierstrass point on $M$ that lies on the same side of $\alpha$ as $\beta$ and is missed by $\beta$.

Summing all the six resulting Weierstrass identities, we then have

$$
\sum 2 \tan ^{-1} \exp \left(-\frac{|\alpha|}{4}-\frac{|\beta|}{2}\right)=\frac{6 \pi}{2},
$$

where the sum is taken over all ordered pairs $(\alpha, \beta)$ of disjoint simple closed geodesics on $M$ such that $\alpha$ is separating and $\beta$ is non-separating.

q.e.d.

Proof of Addendum 1.15. We first prove that the series in (1.18) converges absolutely and uniformly on compact sets in the space $\mathcal{Q F}$ of quasi-Fuchsian representations of $\pi_{1}(M)$ into $\operatorname{PSL}(2, \mathbf{C})$ by the same argument as used in [2]. The identity (1.18) then follows by analytic continuation since (a) each summand in it is an analytic function of the complex Fenchel-Nielsen coordinates for the quasi-Fuchsian space (see $[\mathbf{2 7}]$ ), (b) the identity holds when all the coordinates take real values (by Theorem 1.13) and (c) the space of quasi-Fuchsian representations of $\pi_{1}(M)$ into $\operatorname{PSL}(2, \mathbf{C})$ is simply connected. 
As pointed out in [2] Lemma 5.2, by [16] Lemma 3, for any compact subset $\mathcal{C}$ of $\mathcal{Q} \mathcal{F}$, there is a constant $k=k(\mathcal{C})>0$ such that

$$
k l_{\rho_{0}}(\gamma) \leq \Re l_{\rho}(\gamma) \leq k^{-1} l_{\rho_{0}}(\gamma),
$$

for all $\rho \in \mathcal{C}$ and for all essential simple closed curves $\gamma$, where $\rho_{0}$ is a fixed Fuchsian representation of $\pi_{1}(M)$ into $\operatorname{PSL}(2, \mathbf{C})$.

Since $\left|\tan ^{-1}(z)\right| \leq 2|z|$ for $|z|$ sufficiently small (actually, it is enough if $|z| \leq \sqrt{2} / 2$ ), we have for all except a finite number of pairs of (free homotopy classes up to inversion of) disjoint essential simple closed curves $\alpha, \beta$ on $M$ such that $\alpha$ is separating and $\beta$ is non-separating and for all $\rho \in \mathcal{C}$

$$
\begin{aligned}
\left|\tan ^{-1} \exp \left(-\frac{l_{\rho}(\alpha)}{4}-\frac{l_{\rho}(\beta)}{2}\right)\right| & \leq 2\left|\exp \left(-\frac{l_{\rho}(\alpha)}{4}-\frac{l_{\rho}(\beta)}{2}\right)\right| \\
& =2 \exp \left(-\frac{\Re l_{\rho}(\alpha)}{4}-\frac{\Re l_{\rho}(\beta)}{2}\right) \\
& \leq 2 \exp \left(-k\left(\frac{l_{\rho_{0}}(\alpha)}{4}+\frac{l_{\rho_{0}}(\beta)}{2}\right)\right) .
\end{aligned}
$$

Thus, the series in (1.18) converges absolutely and uniformly on the given compact set $\mathcal{C}$ of $\mathcal{Q} \mathcal{F}$ since the series

$$
\sum \exp \left(-k\left(\frac{l_{\rho_{0}}(\alpha)}{4}+\frac{l_{\rho_{0}}(\beta)}{2}\right)\right)
$$

converges by Lemma 8.5.

q.e.d.

\section{Complexified reformulation of the generalized McShane's identity}

In this section, we prove the unified version (1.22) of our generalized McShane's identity using complex arguments and interpret it geometrically.

Two functions. First, we would like to define two functions $G, S$ : $\mathbf{C}^{3} \rightarrow \mathbf{C}$ as follows:

$$
\begin{aligned}
& G(x, y, z)=2 \tanh ^{-1}\left(\frac{\sinh (x)}{\cosh (x)+\exp (y+z)}\right), \\
& S(x, y, z)=\tanh ^{-1}\left(\frac{\sinh (x) \sinh (y)}{\cosh (z)+\cosh (x) \cosh (y)}\right) .
\end{aligned}
$$

Here, the function $\tanh ^{-1}$ is defined by (1.19), i.e., for a complex number $u \neq \pm 1, \tanh ^{-1} u$ is defined to have imaginary part in $(-\pi / 2, \pi / 2]$ by

$$
\tanh ^{-1} u=\frac{1}{2} \log \frac{1+u}{1-u},
$$


where $\log (\cdot)$ assumes its main branch value, i.e., with imaginary part in $(-\pi, \pi]$. Using this, it is easy to see that the two functions have also the following expressions:

$$
\begin{aligned}
G(x, y, z) & =\log \frac{\exp (x)+\exp (y+z)}{\exp (-x)+\exp (y+z)} \\
S(x, y, z) & =\frac{1}{2} \log \frac{\cosh (z)+\cosh (x+y)}{\cosh (z)+\cosh (x-y)}
\end{aligned}
$$

as used by Mirzakhani in [23]. (She uses different notations $\mathcal{D}, \mathcal{R}$ as explained below.) Here, for a non-zero complex number $u, \log (u)$ assumes its main branch value, i.e., with imaginary part in $(-\pi, \pi]$. We shall see that both expressions of the functions are useful.

For $x, y, z>0$, the geometrical meanings of $G(x, y, z)$ and $S(x, y, z)$ are as follows. Let $\mathcal{P}(2 x, 2 y, 2 z)$ be the unique (up to isometry) hyperbolic pair of pants whose boundary components $X, Y, Z$ are simple closed geodesics of lengths $2 x, 2 y, 2 z$ respectively. Then, $S(x, y, z)$ is half the length of the orthogonal projection of the boundary geodesic $Y$ onto $X$ in $\mathcal{P}(2 x, 2 y, 2 z)$ and $S(x, z, y)$ is half the length of the orthogonal projection of the boundary geodesic $Z$ onto $X$ in $\mathcal{P}(2 x, 2 y, 2 z)$, while $G(x, y, z)$ is the length of each of the two gaps between these two projections on $X$. We have therefore the identity

$$
G(x, y, z)+S(x, y, z)+S(x, z, y)=x
$$

for all $x, y, z \geq 0$. Note that the same identity holds modulo $\pi i$ for all $x, y, z \in \mathbf{C}$.

Remark 10.1. The relations of our functions $G, S$ with Mirzakhani's functions $\mathcal{D}, \mathcal{R}$ are

$$
\begin{aligned}
G(x, y, z) & =\mathcal{D}(2 x, 2 y, 2 z) / 2 \\
S(x, y, z) & =x-\mathcal{R}(2 x, 2 z, 2 y) / 2 .
\end{aligned}
$$

The following lemma gives a mild extension of (10.5), without modulo $\pi i$, which will be needed in the proof of Theorem 10.3 below. 


\section{Lemma 10.2.}

(i) For $x, z \geq 0$ and $y \in\left[0, \frac{\pi}{2}\right]$,

$$
\begin{aligned}
& G(x, y i, z)+S(x, y i, z) \\
& =x-\tanh ^{-1}\left(\frac{\sinh (x) \sinh (z)}{\cos (y)+\cosh (x) \cosh (z)}\right) .
\end{aligned}
$$

(ii) For $x, y \in\left[0, \frac{\pi}{2}\right]$ and $z \geq 0$,

$$
\begin{aligned}
& G(x i, y i, z)+S(x i, y i, z) \\
& =\left[x-\tan ^{-1}\left(\frac{\sin (x) \sinh (z)}{\cos (y)+\cos (x) \cosh (z)}\right)\right] i .
\end{aligned}
$$

The proof we have is a messy but elementary calculation; details can be found in [30] Section 2.2.

Restatement of the complexified identities. Now, we can restate the non-cusp cases of Theorem 1.16 using the functions $G, S$ defined above. Recall that for each generalized simple closed geodesic $\delta$, we have defined in Section 1 its complex length $|\delta|$, that is, $|\delta|=0$ if $\delta$ is a cusp; $|\delta|=\theta i$ if $\delta$ is a cone point of angle $\theta \in(0, \pi]$; and $|\delta|=l$ if $\delta$ is a boundary geodesic or an interior generalized simple closed geodesic of hyperbolic length $l>0$.

Theorem 10.3. For a compact hyperbolic cone-surface $M$ with all cone angles in $(0, \pi]$, let all its geometric boundary components be $\Delta_{0}$, $\Delta_{1}, \ldots, \Delta_{n}$ with complex lengths $L_{0}, L_{1}, \ldots, L_{n}$ respectively. If $\Delta_{0}$ is a cone point or a boundary geodesic then

$$
\sum_{\alpha, \beta} G\left(\frac{L_{0}}{2}, \frac{|\alpha|}{2}, \frac{|\beta|}{2}\right)+\sum_{j=1}^{n} \sum_{\beta} S\left(\frac{L_{0}}{2}, \frac{L_{j}}{2}, \frac{|\beta|}{2}\right)=\frac{L_{0}}{2},
$$

where the first sum is taken over all unordered pairs of generalized simple closed geodesics $\alpha, \beta$ on $M$ such that $\alpha, \beta$ bound with $\Delta_{0}$ an embedded pair of pants on $M$ (note that one of $\alpha, \beta$ might be a geometric boundary component) and the sub-sum in the second sum is taken over all interior simple closed geodesics $\beta$ such that $\beta$ bounds with $\Delta_{j}$ and $\Delta_{0}$ an embedded pair of pants on $M$. Furthermore, all the series in (10.10) converge absolutely.

Remark 10.4. We shall omit the proof of Theorem 1.16 in the case where $\Delta_{0}$ is a cusp, for as remarked before, in the cusp case, the identity (1.23) can either be proved similarly or be derived by considering the first order infinitesimal terms of the corresponding identity (1.22) in other cases.

Proof. We first show that our generalized McShane's identities (1.8) and (1.9) can be reformulated as (10.10) modulo convergence. 
First, suppose that $\Delta_{0}$ is a boundary geodesic of hyperbolic length $l_{0}>0$.

For a pair of interior generalized simple closed geodesics $\alpha, \beta$ which bound with $\Delta_{0}$ an embedded pair of pants on $M$, we have directly by definition that

$$
\operatorname{Gap}\left(\Delta_{0} ; \alpha, \beta\right)=G\left(\frac{l_{0}}{2}, \frac{|\alpha|}{2}, \frac{|\beta|}{2}\right) .
$$

For a pair of generalized simple closed geodesics $\alpha, \beta$ such that $\alpha$ is a boundary geodesic and $\beta$ is an interior generalized simple closed geodesic and that they bound with $\Delta_{0}$ an embedded pair of pants on $M$, we have by definition and the geometric meanings of $G, S$ that

$$
\operatorname{Gap}\left(\Delta_{0} ; \alpha, \beta\right)=G\left(\frac{l_{0}}{2}, \frac{|\alpha|}{2}, \frac{|\beta|}{2}\right)+S\left(\frac{l_{0}}{2}, \frac{|\alpha|}{2}, \frac{|\beta|}{2}\right) .
$$

For a pair of generalized simple closed geodesics $\alpha, \beta$ such that $\alpha$ is a cone point of angle $\varphi \in(0, \pi]$ and $\beta$ is an interior generalized simple closed geodesic and that they bound with $\Delta_{0}$ an embedded pair of pants on $M$, we have by (10.8) with $x=l_{0} / 2, y=\varphi / 2, z=|\beta| / 2$ that

$$
\operatorname{Gap}\left(\Delta_{0} ; \alpha, \beta\right)=G\left(\frac{l_{0}}{2}, \frac{\varphi i}{2}, \frac{|\beta|}{2}\right)+S\left(\frac{l_{0}}{2}, \frac{\varphi i}{2}, \frac{|\beta|}{2}\right) .
$$

Next, suppose that $\Delta_{0}$ is a cone point of angle $\theta_{0} \in(0, \pi]$.

For a pair of interior generalized simple closed geodesics $\alpha, \beta$ which bound with $\Delta_{0}$ an embedded pair of pants on $M$, we have by definition that

$$
\operatorname{Gap}\left(\Delta_{0} ; \alpha, \beta\right) i=G\left(\frac{\theta_{0} i}{2}, \frac{|\alpha|}{2}, \frac{|\beta|}{2}\right) .
$$

For a pair of generalized simple closed geodesics $\alpha, \beta$ such that $\alpha$ is a boundary geodesic and $\beta$ is an interior generalized simple closed geodesic and that they bound with $\Delta_{0}$ an embedded pair of pants on $M$, we have by the analysis in Section 7 that

$$
\begin{aligned}
& \operatorname{Gap}\left(\Delta_{0} ; \alpha, \beta\right) i \\
& =2 i \tan ^{-1} \frac{\sin \frac{\theta_{0}}{2}}{\cos \frac{\theta_{0}}{2}+\exp \frac{|\alpha|+|\beta|}{2}}+i \tan ^{-1} \frac{\sin \frac{\theta_{0}}{2} \sinh \frac{|\alpha|}{2}}{\cosh \frac{|\beta|}{2}+\cos \frac{\theta_{0}}{2} \cosh \frac{|\alpha|}{2}} \\
& \quad=G\left(\frac{\theta_{0} i}{2}, \frac{|\alpha|}{2}, \frac{|\beta|}{2}\right)+S\left(\frac{\theta_{0} i}{2}, \frac{|\alpha|}{2}, \frac{|\beta|}{2}\right) .
\end{aligned}
$$

For a pair of generalized simple closed geodesics $\alpha, \beta$ such that $\alpha$ is a cone point of angle $\varphi \in(0, \pi]$ and $\beta$ is an interior generalized simple closed geodesic and that they bound with $\Delta_{0}$ an embedded pair of pants 
on $M$, we have by (10.9) with $x=\theta_{0} / 2, y=\varphi / 2, z=|\beta| / 2$ that

$$
\operatorname{Gap}\left(\Delta_{0} ; \alpha, \beta\right) i=G\left(\frac{\theta_{0} i}{2}, \frac{\varphi i}{2}, \frac{|\beta|}{2}\right)+S\left(\frac{\theta_{0} i}{2}, \frac{\varphi i}{2}, \frac{|\beta|}{2}\right) .
$$

Finally, we prove the absolute convergence of the series in (10.10). It is not hard to see from the above analysis that we only need to prove, for each $j=1, \ldots, n$, the absolute convergence of the series

$$
\sum_{\beta} S\left(\frac{L_{0}}{2}, \frac{L_{j}}{2}, \frac{|\beta|}{2}\right),
$$

where the sum is over all interior generalized simple closed geodesics $\beta$ which bounds with $\Delta_{j}$ and $\Delta_{0}$ an embedded pair of pants on $M$. The desired absolute convergence follows from Lemma 8.5 since

$$
S\left(\frac{L_{0}}{2}, \frac{L_{j}}{2}, \frac{|\beta|}{2}\right) \sim \frac{\sinh \frac{L_{0}}{2} \sinh \frac{L_{j}}{2}}{\cosh \frac{|\beta|}{2}} \sim \text { const. } \exp \left(-\frac{|\beta|}{2}\right)
$$

as $|\beta| \rightarrow \infty$ and there are only a finite number of interior generalized simple closed geodesics $\beta$ with $|\beta| \leq c$ for any give $c>0$. $\quad$ q.e.d.

Geometric interpretation. We would like to explore the geometric meanings of the summands in the complexified formula (10.10). As noted earlier, because of the complexification, the summands are no longer necessarily real and positive, and so no longer admit interpretations as simply gaps on the cone-surface. Indeed, one really needs to think of the cone surface as being developed onto a totally geodesic hyperplane in $\mathbb{H}^{3}$ to interpret the summands properly.

In the case that $M$ has no cone points, all its geometric boundary components (here cusps are treated as boundary geodesics of length 0 ) $\Delta_{0}, \Delta_{1}, \ldots, \Delta_{n}$ are boundary geodesics with hyperbolic lengths $L_{0}, L_{1}$, $\ldots, L_{n}$ respectively. Assume $\Delta_{0}$ is not a cusp, that is, $L_{0}>0$. Then as explained in Section 7, in the first sum, the summand is the width of one of the main gaps in the pair of pants $\mathcal{P}\left(\Delta_{0}, \alpha, \beta\right)$ bounded by $\Delta_{0}$ and $\alpha, \beta$; while in the second sum, the sub-summand is the width of one of the two extra gaps associated to $\Delta_{j}$ in the pair of pants $\mathcal{P}\left(\Delta_{0}, \Delta_{j}, \beta\right)$ bounded by $\Delta_{0}, \Delta_{j}$ and $\beta$. We would like to think of the union of the two extra gaps in $\mathcal{P}\left(\Delta_{0}, \Delta_{j}, \beta\right)$ as the orthogonal projection of $\Delta_{j}$ onto $\Delta_{0}$ along the common perpendicular $\delta$ of $\Delta_{j}$ and $\Delta_{0}$ in $\mathcal{P}\left(\Delta_{0}, \Delta_{j}, \beta\right)$ and think of its width as the direct visual measure of $\Delta_{j}$ seen at $\Delta_{0}$ along $\delta$. Hence, the second part of the left-hand side of (10.10) can be thought of as the total direct visual measure of all the non-distinguished geometric boundary components $\Delta_{1}, \ldots, \Delta_{n}$ seen at $\Delta_{0}$.

In the case that $\Delta_{0}$ is a cone point of angle $\theta_{0} \in(0, \pi]$ (hence $L_{0}=$ $\left.\theta_{0} i\right)$ and all other geometric boundary components of $M$ are boundary geodesics (here, cusps treated as boundary geodesics of length 0 ), for 
each pair of generalized simple closed geodesics $\alpha, \beta$ which bound with $\Delta_{0}$ an embedded pair of pants $\mathcal{P}\left(\Delta_{0}, \alpha, \beta\right)$ on $M$, each of $\alpha, \beta$ has a direct visual angle at the cone point $\Delta_{0}$; and the summand in the first sum is $i$ times the angle measure of one of the two gaps at $\Delta_{0}$ between the two $\Delta_{0}$-geodesic rays asymptotic to $\alpha^{+}, \beta^{-}$(respectively $\alpha^{-}, \beta^{+}$). The sub-summand in the second sum is $i$ times half the visual angle measure of $\Delta_{j}$ at $\Delta_{0}$ in the pair of pants $\mathcal{P}\left(\Delta_{0}, \Delta_{j}, \beta\right)$ on $M$.

If $M$ has cone points other than $\Delta_{0}$, the formulations of the generalized McShane's identities (1.8)-(1.10) in terms of $\operatorname{Gap}\left(\Delta_{0} ; \alpha, \beta\right)$ will not be as neat as in the two special cases above. The problem lies in that a cone point (other than $\Delta_{0}$ ) seems to have direct visual measure zero at $\Delta_{0}$, causing the formulae to be non-uniform. However, this non-uniformity is caused by the (wrong) point of view that we treat a cone point as only a point. The correct point of view is (perhaps) that a cone point (as a geometric boundary component) should be a geodesic axis perpendicular to the surface at the very cone point when the surface is developed into hyperbolic 3 -space and hence, one should use purely imaginary instead of real length for a cone point. Thus, the visual measure of a cone point $\Delta_{j}, j \neq 0$ should be the visual measure of the geodesic axis, and is in fact purely imaginary if $\Delta_{0}$ is a boundary geodesic and $\Delta_{j}$ a cone point! Similarly, the main gaps will now be measured using the end points of the geodesic axis of the cone point rather than the cone point itself, and will be complex rather than real. The functions $G(x, y, z)$ and $S(x, y, z)$ are then really measuring the gaps according to this point of view, see [30] Section 2.5 for details. This interpretation is also exploited in our generalization of the identity to classical Schottky groups in [29]. More generally, the point of view of using complex translation length for an isometry of the hyperbolic 3 -space is well discussed in details in $[\mathbf{1 2}]$ and $[\mathbf{2 6}]$.

\section{References}

[1] H. Akiyoshi, H. Miyachi \& M. Sakuma, A refinement of McShane's identity for quasifuchsian punctured torus groups, in 'The Tradition of Ahlfors and Bers, III', The Ahlfors-Bers Colloquium, Oct. 2001, Univ. of Connecticut at Storrs, W. Abikoff, A. Haas (Eds.), Contemporary Mathematics, 355, 21-40, American Mathematical Society, 2004, MR 2145054.

[2] H. Akiyoshi, H. Miyachi \& M. Sakuma, Variations of McShane's identity for punctured surface groups, London Mathematical Society Lecture Notes, Y. Minsky, M. Sakuma \& C. Series (Eds.), Cambridge University Press, to appear.

[3] A. Basmajian, The orthogonal spectrum of a hyperbolic manifold, Amer. J. Math. 115(5) (1993) 1139-1159, MR 1246187, Zbl 0794.30032.

[4] A.F. Beardon, The Geometry of Discrete Groups, Graduate Texts in Mathematics, 91, Springer-Verlag, New York, 1983, MR 1393195, Zbl 0528.30001.

[5] L. Bers, Uniformization, moduli and Kleinian groups, Bull. London Math. Soc. 4 (1972) 257-300, MR 0348097, Zbl 0257.32012. 
[6] J.S. Birman \& C.M. Series, Geodesics with bounded intersection number on surfaces are sparsely distributed, Topology 24(2) (1985) 217-225, MR 0793185, Zbl 0568.57006.

[7] B.H. Bowditch, A proof of McShane's identity via Markoff triples, Bull. London Math. Soc. 28(1) (1996) 73-78, MR 1356829, Zbl 0854.57009.

[8] B.H. Bowditch, A variation of McShane's identity for once-punctured torus bundles, Topology 36(2) (1997) 325-334, MR 1415591, Zbl 0872.57015.

[9] B.H. Bowditch, Markoff triples and quasi-Fuchsian groups, Proc. London Math. Soc. (3) 77(3) (1998) 697-736, MR 1643429, Zbl 0928.11030.

[10] P. Buser, Geometry and Spectra of Compact Riemann Surfaces, Birkhäuser, Boston, 1992, MR 1183224, Zbl 0770.53001.

[11] D. Cooper, C.D. Hodgson \& S.P. Kerckhoff, Three-dimensional orbifolds and cone-manifolds, MSJ Memoirs, 5, Mathematical Society of Japan, Tokyo, 2000, MR 1778789, Zbl 0955.57014.

[12] W. Fenchel, Elementary Geometry in Hyperbolic Space, Walter de Gruyter \& Co., Berlin, 1989, MR 1004006, Zbl 0674.51001.

[13] W.M. Goldman, The modular group action on real $\mathrm{SL}(2)$-characters of a oneholed torus, Geom. Topol. 7 (2003) 443-486, MR 2026539, Zbl 1037.57001.

[14] A. Haas, Diophantine approximation on hyperbolic Riemann surfaces, Acta Math. 156(1-2) (1986) 33-82, MR 0822330, Zbl 0593.10028.

[15] A. Haas \& P. Susskind, The geometry of the hyperelliptic involution in genus two, Proc. Amer. Math. Soc. 105(1) (1989) 159-165, MR 0930247, Zbl 0672.30033.

[16] T. Jørgensen \& A. Marden, Two doubly degenerate groups, Quart. J. Math. Oxford (2) 30(118) (1979) 143-156, MR 0534829, Zbl 0414.30035.

[17] A. Marden, The geometry of finitely generated Kleinian groups, Ann. of Math. 99 (1974) 383-462, MR 0349992, Zbl 0282.30014.

[18] G. McShane, A remarkable identity for lengths of curves, Ph.D. Thesis, University of Warwick, 1991.

[19] G. McShane, Simple geodesics and a series constant over Teichmuller space, Invent. Math. 132(3) (1998) 607-632, MR 1625712, Zbl 0916.30039.

[20] G. McShane, Weierstrass points and simple geodesics, Bull. London Math. Soc. 36(2) (2004) 181-187, MR 2026411, Zbl 1052.57021.

[21] G. McShane, Length series on Teichmuller space, arXiv:math.GT/0403041.

[22] G. McShane, Simple geodesics on surfaces of genus 2, preprint.

[23] M. Mirzakhani, Simple geodesics and Weil-Petersson volumes of moduli spaces of bordered Riemann surfaces, preprint.

[24] M. Sakuma, Variations of McShane's identity for the Riley slice and 2bridge links, in 'Hyperbolic spaces and related topics' (Japanese) (Kyoto, 1998), Surikaisekikenkyusho Kokyuroku 1104 (1999) 103-108, MR 1744474, Zbl 0951.57500.

[25] P.S. Schaller, Geometry of Riemann surfaces based on closed geodesics, Bull. Amer. Math. Soc. (N.S.) 35(3) (1998) 193-214, MR 1609636, Zbl 1012.30029.

[26] C. Series, An extension of Wolpert's derivative formula, Pacific J. Math. 197(1) (2001) 223-239, MR 1810217, Zbl 1065.30044.

[27] S.P. Tan, Complex Fenchel-Nielsen coordinates for quasi-Fuchsian structures, Internat. J. Math. 5(2) (1994) 239-251, MR 1266284, Zbl 0816.32017. 
[28] S.P. Tan, Y.L. Wong \& Y. Zhang, Generalized Markoff maps and McShane's identity, arXiv:math.GT0502464.

[29] S.P. Tan, Y.L. Wong \& Y. Zhang, McShane's identity for classical Schottky groups, arXiv:math. GT0411628.

[30] Y. Zhang, Hyperbolic cone-surfaces, generalized Markoff maps, Schottky groups and McShane's identity, Ph.D. thesis, National University of Singapore, 2004.

Department of Mathematics National University of Singapore 2 SCIENCE DRIVE 2

SINGAPORE 117543

SINGAPORE

E-mail address: mattansp@nus.edu.sg

Department of Mathematics

National University of Singapore 2 Science Drive 2 SINGAPORE 117543

SINGAPORE

E-mail address: matwyl@nus.edu.sg

Department of MATHEMAtics YANGZHOU UNIVERSITY

YANGZHOU, JiAnGSU 225002 P. R. CHINA

E-mail address: yingzhang@yzu.edu.cn 